\author{
胶体光子晶体膜的超浸润性研究进展 \\ $\begin{array}{rlll}\text { 崔丽影 }^{a} & \text { 范莎莎 } & \text { 于存龙 } \\ & { }^{a} \text { 吉林农业大学 } & \text { 资源与环境学院 } & \text { 长春 } 130118)\end{array}$ \\ $\left({ }^{b}\right.$ 中国科学院理化技术研究所 仿生材料与界面科学院重点实验室 北京 100190) \\ ( ${ }^{\mathcal{C}}$ 中国科学院大学未来技术学院 仿生材料与界面教研室 北京 100049)
}

\begin{abstract}
摘要 近年来, 由于具有特殊浸润性的胶体光子晶体膜在传感、检测、催化等方面的重要应用, 胶体光子晶体膜的超 浸润研究受到科研工作者的广泛关注. 本文阐述了包括超亲液、超疏液、两亲性、梯度浸润性、可调控浸润性、图案 浸润性等具有特殊浸润性光子晶体膜的制备及其相关应用，并讨论疏水、超疏水和亲-疏图案不同浸润性基底对所制备 胶体光子晶体膜的功能性及其相关应用的影响. 该工作对于发展新型功能型材料器件的制备具有重要的借鉴及指导意 义.
\end{abstract}

关键词＼cjkstart特殊浸润性；胶体光子晶体；制备；功能性；应用

\title{
Research Progress on the Super-wettability of Colloidal Photonic Crystals
}

\author{
Cui, Liying ${ }^{a} \quad$ Fan, Shasha $^{a} \quad$ Yu, Cunlong ${ }^{a} \quad$ Kuang, Minxuan ${ }^{b} \quad$ Wang, Jingxia*, ${ }^{*, c}$ \\ ( ${ }^{a}$ College of Resources and Environment, Jilin Agricultural University, Changchun 130118) \\ ( ${ }^{b}$ Laboratory of Bioinspired Materials and Interface Science, Technical Institute of Physics and Chemistry, Chinese Academy \\ of Sciences, Beijing 100190) \\ ( ${ }^{c}$ Laboratory of Bioinspired Materials, School of Future Technology, University of Chinese Academy of Sciences, Beijing \\ 100049)
}

\begin{abstract}
In recent years, the wettability of colloidal PCs has attracted much interest from researchers due to potential applications in printing, sensor, microfluidics and so on. In this paper, we present two kinds of research work related to PCs' wettability. On the one hand, the functional colloidal PCs have been fabricated from the modification of its wettability. Where, the wettability of PCs can be modified from superhydrophilic, superhydrophobic, amphiphilic, gradient wettability, controllable wettability and patterned wettability. Wettability is an important property of solid surface and can be generally controlled mainly by its surface chemical composition and surface topographic structure. Surface chemical composition determines surface free energy (i.e., hydrophilicity/hydrophobicity), while the surface topographic structure can amplify hydrophilicity or hydrophobicity, based on the Wenzel and modified Cassie equation. Thus, PCs with specific wettability have been fabricated based on their intrinsic, well-ordered surface topographic structure, and chemical composition. The superhydrophilic and superhydrophobic PCs have been achieved based on the amplification effect of the surface well-ordered topographic structure. The gradient PCs have been fabricated by changing the topographic structure. The PCs with controllable wettability can be obtained when introducing a responsive group onto PCs' surface. The underwater oil-adhesion properties of PCs have been controlled by varying the latex from spherical or cauliflower-like to single cavity. On the other hand, functional PCs are fabricated from the substrate with specific wettability. Typically, high-quality and crack free PCs are achieved from superhydrophobic substrate, pattern PCs from the hydrophilic-hydrophobic substrate, PC dome with excellent wide-angle property is fabricated from hydrophobic substrate. Otherwise, gas-liquid or liquid-liquid interface has also been included as a special substrate for the fabrication of functional PCs, such as flower-shape or cake-shaped Janus PCs. Colloidal photonic crystals (PCs), the periodic arrangement of monodispersed latex spheres, have attracted much interest from researchers due to their unique light manipulation properties. The combination of the special wettability and light manipulation properties of PCs will bring many novel properties and promising applications. Finally, the outlook and challenges for colloidal photonic crystals with special wettability are discussed. The work is of importance for the creation of novel functional materials.
\end{abstract}

Keywords special wettability; colloidal photonic crystals; preparation; functionality; applications

\footnotetext{
* E-mail: jingxiawang@mail.ipc.ac.cn; Tel.: 010-82543510

Received July 5, 2017; published September 4, 2017.

Project supported by the National Natural Science Foundation of China (Nos. 51403076, 51673207, 51503214, 51373183).

项目受国家自然科学基金(Nos. 51403076, 51673207, 51503214, 51373183)资助.
} 


\section{1 引言}

浸润性是固体表面的重要性质之一, 主要由其表面 化学组成和多尺度微纳结构决定. 1805 年 Young ${ }^{[1]}$ 首次 通过接触角对浸润性进行定义: 认为其主要由基材表面 的化学组成决定, 水接触角接近 $0^{\circ}$ 的表面为超亲水表 面，水接触角大于 $150^{\circ}$ 的表面为超疏水表面. 1936 年 Wenzel $^{[2]}$ 提出一种理论模型阐述固体表面宏观粗粘度和 接触角的关系, 并进一步提出表面粗粘因子这一术语 ${ }^{[3]}$. 1944 年 Cassie 和 Baxter ${ }^{[4]}$ 将 Wenzel 模型进行优化并延 伸到能捕获固体和液体间空气的多孔表面和粗鋉表面. 随后研究者对浸润性的理论和分析技术展开了广泛的 研究. 2002 年中科院化学研究所江雷课题组率先揭示了 荷叶表面的超疏水自清洁效应是源于荷叶表面的微纳 复合结构及其表面蜡质化学组成二元协同结果 ${ }^{[5]}$, 之后 该课题组在具有特殊浸润性仿生界面材料的合成与制 备方面开展了深入的研究 ${ }^{[6-21]}$. 他们将特殊浸润性理念 应用于自清洁 ${ }^{[5,9]}$ 、集水 ${ }^{[6,11]}$ 、防雾 ${ }^{[10]}$ 、油水分离 ${ }^{[7,8]}$ 、智

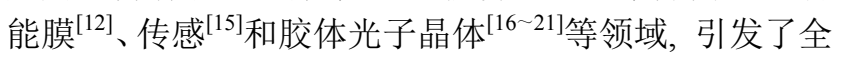
世界学者的浸润性研究热潮.

胶体光子晶体是指直径在微米或亚微米级别的无 机或有机单分散乳胶粒在重力、静电力或毛细管力等作 用下组装形成的二维或三维有序阵列结构. 当胶体晶体 的晶格在可见光范围发生布拉格衍射时会呈现不同的 颜色. 光子晶体特殊的周期结构、大的比表面积、独特 的光调控性能及在新颖光学器件等领域的潜在应用前 景使其在基础研究及实际应用方面吸引了科研人员的 广泛关注. 通常固体表面的化学组成决定其表面自由 能, 从而决定表面的亲水性或疏水性. 依据 Wenzel 和改 进的 Cassie 方程, 光子晶体膜表面周期性微纳米结构产 生的粗䊁度可增强其浸润性使其表面更疏水或更亲水. 近十年来, 根据胶体光子晶体的周期有序结构形成粗糙 度对光子晶体浸润性的放大效应促使光子晶体产生了 更多有趣的性能及相关应用, 也为胶体光子晶体的科学

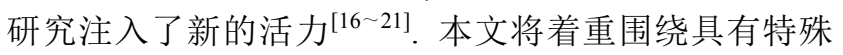
浸润性的胶体光子晶体的制备及其功能性, 以及基底浸 润性对组装功能性胶体光子晶体的影响两方面来介绍 有关胶体光子晶体浸润性的研究进展.

\section{2 具有特殊浸润性的胶体光子晶体膜的制备及 其功能性}

自然界中生物光子晶体为适应不同的生存环境历 经亿万年的进化与演变而产生一些独特的性能, 如: 绚 丽夺目的色彩、特殊的浸润性和耐候性. 受自然界生物 光子晶体的这些特殊性能启发, 具有特殊浸润性的胶体 光子晶体膜的制备及应用受到科研人员的广泛关注. 中 科院理化技术研究所王京霞等 ${ }^{[22]}$ 详细综述了不同浸润 性光子晶体的制备方法及相关应用. 具有特殊浸润性光
子晶体的制备主要是结合胶体光子晶体的本征周期有 序结构及其表面的化学组成. 利用胶体组装结构对亲、 疏水和两亲性材料的浸润性放大作用，实现超亲、超疏 和两亲性胶体光子晶体膜的制备. 通过调控材料的粗粘 结构, 实现具有梯度浸润性胶体光子晶体膜的制备. 通 过将响应材料引入到光子晶体体系中, 实现具有可调控 浸润性胶体光子晶体膜的制备. 另外, 通过单腔、多腔、 菜花型等各向异性乳胶粒的合成及组装可实现水下可 控油粘附性胶体光子晶体膜的制备 ${ }^{[23]}$. 这些不同浸润 性光子晶体的制备对于发展材料的不同应用具有重要 意义. 比如, 超亲液的光子晶体有利于快速灵敏的传感 检测, 超疏液的光子晶体有利于其自清洁及防污. 本节 会详细讨论不同浸润性光子晶体的制备及应用.

\section{1 超亲液的胶体光子晶体膜}

超亲液胶体光子晶体包括超亲水和超亲油胶体光 子晶体. 自然界中存在许多亲水性光子晶体, 例如: 珍 珠和非洲的天牛甲虫的鞘翅. 珍珠是一维光子晶体, 其 亲水性主要是源于组装材料碳酸钙的亲水性 ${ }^{[24]}$. 由于 珍珠形成过程完全在水环境中进行, 适当的亲水性有利 于其不断的吸收水体系中的有机物质而长大. 非洲天牛 甲虫鞘翅表面的鳞片有序排列形成二维光子晶体结构, 该结构表面的亲水性也是源于组成材料的亲水性 ${ }^{[25]}$. 同时, 表面的亲水性使之能随外界湿度的变化而改变颜 色, 使其能与所在环境的背景颜色相一致, 以实现保护 色的效果. $\mathrm{TiO}_{2}$ 作为 “明星” 材料不仅具有光催化性能, 而且还具有光诱导超亲水性能, 这使其在工业和日常生 活中得到广泛应用. 2004 年东南大学顾忠泽课题组 ${ }^{[26]}$ 利 用模版法制备了超亲水的三维反蛋白石结构 $\mathrm{TiO}_{2}$ 光子 晶体膜, 其水接触角是 $0^{\circ}$, 该膜具有亮丽的结构色彩、 自清洁和超亲水性能. 相较通常 $\mathrm{TiO}_{2}$ 膜的超亲水性只 保持大约 3 个月，该法制备的 $\mathrm{TiO}_{2}$ 多孔膜的超亲水性可 保持一年以上. 胶体光子晶体的超亲水性还可以通过多 尺度结构实现, 2011 年中科院固体物理研究所李越课题 组利用二元胶体光子晶体和脉冲激光沉积制备得到微 米、亚微米和纳米复杂分级结构胶体光子晶体, 其不需 要特殊处理就可以实现超亲水性, 该结构的接触角为 $5.2^{\circ[27]}$. 通过在该结构表面引入金层或利用脉冲激光沉 积 $\mathrm{ZnO}, \mathrm{Fe}_{2} \mathrm{O}_{3}, \mathrm{TiO}_{2}, \mathrm{NiO}, \mathrm{WO}_{3}, \mathrm{SnO}_{2}$ 和 C 实现多种材料 复杂分级结构的制备 ${ }^{[27]}$, 该结构可以应用于表面提高 拉曼散射、生物传感和微流体等领域. 亲水光子晶体广 泛用于湿度传感检测体系. 通过在胶体光子晶体间隙注 入亲水材料可得到亲水光子晶体, 所用的亲水材料包括 聚丙烯酸 ${ }^{[28]}$ 、聚丙烯酰胺 ${ }^{[29,30]}$ 、聚乙二醇 ${ }^{[31]}$ 、聚离子液 体 ${ }^{[32,33]}$ 和聚异丙基丙烯酰胺 ${ }^{[34]}$. 所制备材料的特殊浸润 性对与溶液相关的检测速度和检测范围有着重要的影 响. 2012 年中科院化学研究所宋延林课题组利用喷墨打 印技术制备了尺寸为 $20 \mu \mathrm{m}$ 的光子晶体微滴, 并结合聚 异丙基丙烯酰胺的浸润性转变实现了在不到 $1 \mathrm{~s}$ 时间内 
的快速湿度检测 ${ }^{[34]}$. 类似的超亲油胶体光子晶体可以 用于辨别油品种类 ${ }^{[35,36]}$. 2008 年该课题组通过牺牲模板 法制备得到了聚酚醛树脂光子晶体. 由于聚酚醛树脂具 有很好的油吸附性能和耐受性, 因此, 所制备的光子晶 体具有超亲油性, 基于膜的光学信号与油品的折光指数 之间的对应关系可以用该膜进行油品检测, 并且作为油 检测载体反复使用 ${ }^{[23]}$. 通过选用亲油、高折光指数的碳 材料制备光子晶体, 可实现油品的裸眼可视检测 [36].

\section{2 超疏液的胶体光子晶体膜}

超疏液的胶体光子晶体包括超疏水和超疏油胶体 光子晶体. 超疏水光子晶体的制备方法是在构筑粗䊁结 构的表面涂覆低表面能分子(如氟硅烷), 或者是利用粗 粘结构对疏水材料浸润性的放大作用实现. 2003 年东南 大学顾忠泽课题组借助光子晶体表面的粗糙结构和低 表面自由能的氟硅烷分子实现了超疏水光子晶体膜的 制备. 该方法是利用聚苯乙烯乳胶球和二氧化硅纳米粒 子共沉积法制得三维反蛋白石结构二氧化硅光子晶体, 然后采用热化学气相沉积法对表面进行氟硅烷处理 ${ }^{[37]}$, 该膜不仅产生了类似蝴蝶的亮丽色彩, 还呈现类荷叶的 超疏水自清洁性能. 随后, 科研人员基于二维胶体单层, 通过控制刻蚀时间调节模板球尺寸或基底硅柱阵列尺 寸, 以调节固液接触面积, 从而实现超疏水二维光子晶 体的制备 ${ }^{[38,39]}$. 另外通过在单粒径组装的二维光子晶体 基础上引入纳米结构, 基于微纳复合结构对浸润性放大 效应实现超疏水光子晶体的制备 ${ }^{[40 ~ 49]}$. 典型的例子是, 2007 年中科院化学研究所高雪峰等 ${ }^{[45]}$ 报道了受蚊子复 眼结构启发并结合软印刷方法在微米尺寸聚二甲基硅 氧烷半球表面构筑单层二氧化硅纳米球的有序阵列(图 1a), 通过对其表面进行氟硅烷修饰, 其膜的接触角为 $155^{\circ}$, 最终实现膜的超疏水性能及防雾性能. 超疏水光 子晶体还可以通过全息技术制备 ${ }^{[50 \sim 52]}$. 2012 年宾夕法 尼亚大学杨树课题组 ${ }^{[51]}$ 利用全息刻蚀制得 $3 \mathrm{D}$ 金刚石结 构光子晶体(图 1b), 所选用的材料是环氧功能化的环己 基多面体齐聚倍半硅氧烷，材料表面的纳米粗糙度 $(\leqslant$ $120 \mathrm{~nm}$ ) 是由于冲洗过程中高分子链微相分离导致, 并 且可以通过高分子交联密度调控. 这种可控纳米粗䊁度 的 3D 光子晶体结构可广泛应用于染料敏化太阳能电 池、量子点太阳能电池、燃料电池、催化和蛋白/药物输 送等领域. 2017 年吉林大学孙洪波课题组 ${ }^{[52]}$ 也利用二束 激光干涉刻蚀快速制得仿生分级图案(图 1c). 该结构具 有超疏水、彩虹色、反射方向依赖性光学性能等特点.

由于有机液体的表面张力要比水低得多, 因此, 超 疏油光子晶体表面的制备非常具有挑战性. 2007 年美国 麻省理工学院 Cohen 将凹角(re-entrant)结构与表面化学 组成相结合成功实现超疏油表面的制备 ${ }^{[53,54]}$. 在此基础 上, 2011 年希腊德谟克利特国家科学研究中心 Gogolides 等 ${ }^{[55]}$ 利用这种凹角结构在聚甲基丙烯酸甲酯 高分子表面实现双疏或双亲光子晶体的制备, 凹角结构

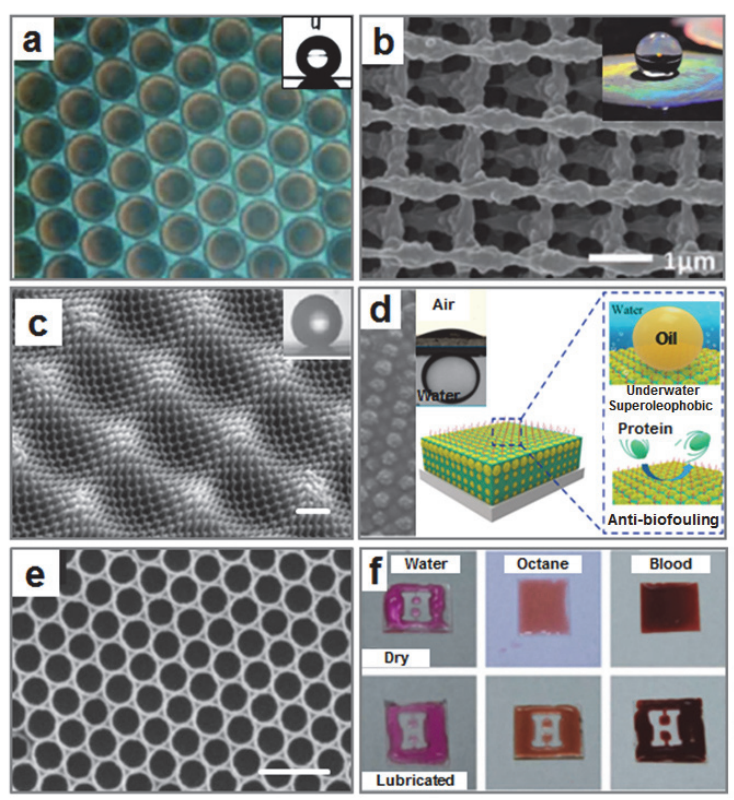

图 1 (a)软刻蚀制备的人工复眼结构 ${ }^{[45]}$; (b)全息制备的金刚石结构光 子晶体 ${ }^{[51]}$; (c) 双束激光干涉刻蚀制备的分级三维结构 ${ }^{[52]}$, 标尺: $1 \mu \mathrm{m}$; (d) 分级微凝胶球组装结构的水下超疏油和防污性能 ${ }^{[57]}$; (e, f) 分别是单 层反蛋白石结构的 SEM 图和抗粘附反蛋白石结构减少各种液体污染 物的吸附 ${ }^{[58]}$, 标尺: $500 \mathrm{~nm}$

Figure 1 (a) The artificial compound-eye analogue prepared by soft lithography ${ }^{[45]}$. (b) The diamond photonic crystals fabricated by holographic lithography ${ }^{[51]}$. (c) The hierarchical 3D textures prepared by two-beam laser interference ${ }^{[52]}$, scale bar: $1 \mu \mathrm{m}$. (d) The assembly structure of hierarchical microgel spheres with underwater superoleophobic and antibiofouling properties ${ }^{[57]}$. (e,f) The SEM image of inverse opal structure and its lubricated state for reducing the absorption of various liquid contaminants ${ }^{[58]}$. Scale bar: $500 \mathrm{~nm}$

是通过胶体刻蚀结合等离子体刻蚀制得. 水下超疏油表 面是指将膜材料置于水相中, 该材料免受水中油的粘 附, 对油的接触角大于 $150^{\circ}$. 该表面对于防止水下材料 免受石油泄漏的污染具有重要意义. 中科院化学所刘明 杰的研究工作 ${ }^{[56]}$ 对于水下超疏油的潜在应用给出了很 好的诠释: 研究发现水中的鱼皮具有类水凝胶结构, 可 以免受油的污染而幸存于石油泄漏. 受此启发, 他制备 了水下超疏油的水凝胶结构, 并对其机理进行了详尽的 分析和讨论 ${ }^{[56]}$. 2016 年复旦大学武利民课题组 ${ }^{[57]}$ 利用分 级结构微凝胶球组装实现水下可修复超疏油防污涂层 的制备 (图 1d). 微凝胶球先通过沉淀聚合合成, 并结合 $\mathrm{SiO}_{2}$ 纳米粒子制得分级结构微凝胶球, 接着在分级结构 表面嫁接亲水的嵌段共聚物, 然后将这些微凝胶球在玻 璃基底自组装, 并在其表面旋涂热塑性丙烯酸树脂、 $80{ }^{\circ} \mathrm{C}$ 固化后制得水下可修复超疏油防污涂层. 在空气 中涂层表面的水接触角为 $31.2^{\circ}$, 水下对十六烷接触角 为 $160.8^{\circ}$, 并且在水下对已烷、庚烷、癸烷、液体石蜡、 石油醚和三氟甲基丙烯酸乙酯等油性液体具有排斥性. 该膜在高酸、碱和盐环境中仍具有疏油防污性能，一旦 机械损伤还可以自修复. 该涂层具有良好的透明性、对 热敏感性强, 可在水下仪器面板、水下指示灯和信号显 示等方面具有潜在应用前景 ${ }^{[57]}$. 将液体引入微纳结构 


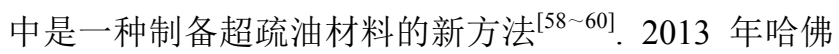
大学 Aizenberg 课题组 ${ }^{[58]}$ 受猪笼草启发利用光滑液体注 入胶体模板法开发出了一种新的透明、可自修复、具有 优异不沾/防垢除垢的材料. 利用胶体模板法可以实现 均一、有序及大面积多孔膜的制备(图 1e), 并且这种方 法可以应用于弯曲和复杂结构基底. 由于润滑剂具有液 体流动性, 因此可以稳定的锁定在结构内, 使表面无缺 陷、可自修复及有效排斥水、油和血液的粘附. 文中使 用的润滑剂为氟化液体, 通过调控表面化学组成也可以 选择其它流体作为润滑剂排斥另一种不溶液体. 进一步 还可以将基底排斥液体这种性能图案化(图 1f), 这将有 利于其在空间限制简单或复杂流体方面的应用. 材料可 耐受强烈的刮擦和捶打测试, 具有很好的耐久性. 这种 材料可以在 $3 \mathrm{D}$ 打印、建造和医学等方面广泛应用.

\section{3 两亲性胶体光子晶体膜}

两亲性胶体光子晶体膜主要是通过两亲性构筑基 元或气液界面制备得到广泛应用于液体输运、传感和微 流体等领域. 2004 年加州大学圣地亚哥分校的 Sailor 课 题组 ${ }^{[61]}$ 分别对多孔硅结构的不同表面进行亲水、亲油的 化学修饰, 再将其超声处理成几十微米的碎片, 并在其 中掺杂磁性纳米粒子. 从而获得两亲性磁调控一维光子 晶体水凝胶颗粒. 在外加磁场作用下, 这些光子晶体水 凝胶颗粒可实现对微升液滴的输运, 并且通过光子晶体 的结构色彩及光学信号对微滴中的反应进行实时监控. 2006 年中科院化学研究所宋延林课题组 ${ }^{[62 ~ 64]}$ 以两亲性 单分散乳胶粒作为胶体晶体的基本构筑单元, 通过对其 表面功能团及核壳结构的设计, 实现了具有特殊浸润性 的胶体光子晶体膜的制备. 其中具有疏水聚苯乙烯硬核 及亲水聚甲基丙烯酸甲酯/聚丙烯酸软壳的单分散乳胶 粒是通过乳液聚合制备得到. 该课题组利用这种两亲性 乳胶粒不仅实现了大面积、图案化胶体光子晶体的制备 和高质量、无裂纹功能性胶体光子晶体的制备; 还将所 制备光子晶体应用于传感、微流体和光学器件等方面. 气液界面胶体球刻蚀法也可以用于制备两亲性光子晶 体, 这是通过漂浮在液面上的单层胶体晶体为模板来制 备二维有序纳米结构的方法. 许多工作利用气液界面胶 体球刻蚀法实现二维有序多孔薄膜的可控制备. 2012 年, 大阪大学工程学院的 Fujii 等 ${ }^{[65]}$ 在空气一水界面组装 聚苯乙烯粒子, 并在乳胶粒没入水的一侧包覆聚吡咯薄 层, 得到 Janus 胶体晶体膜, 其中空气侧和没水侧的水 接触角分别为 $93^{\circ}$ 和 $41^{\circ}$. 当将乳胶粒溶剂溶解去除后, 得到了毫微微(femoto) 升级杯型的各向异性光子晶体结 构. 2016 年, 清华大学李广涛等 ${ }^{[66]}$ 采取类似的方法在聚 苯乙烯表面生长可调控用量的聚多巴胺, 制备得到了 Janus 的光子晶体膜及毫微微升级杯状结构, Janus 膜的 空气侧和没水侧的水接触角分别为 $92.5^{\circ}$ 和 $22.0^{\circ}$, 之后 用 $\mathrm{Ag}$ 纳米粒子修饰并用十一烷硫醇处理后, 使 Janus 膜一侧水接触角增加为 $170^{\circ}$, 从而获得两侧分别为超疏
水和亲水的 Janus 膜, 这种膜在限域结晶方面有特殊应 用.

\section{4 梯度浸润性胶体光子晶体膜}

梯度浸润性表面广泛应用于细胞运动性研究、诊 断、纳米摩擦和微流体等领域. 梯度浸润性胶体光子晶 体膜的制备可以通过在平行或垂直膜的方向构建梯度 外场实现. 2005 年中科院长春应用化学研究所韩艳春课 题组 ${ }^{[67]}$ 利用简单的温度梯度场调控聚苯乙烯乳胶球的 形貌，从而实现梯度浸润性胶体光子晶体膜的制备，膜 的浸润性从一端到另一端依次为 $88.7^{\circ}, 108.6^{\circ}, 121.2^{\circ}$, $132.2^{\circ}, 148.1^{\circ}$. 具体制备方法是将乳液聚合制备得到的 聚苯乙烯微球旋涂制得光子晶体膜. 其接触角为 $147.2^{\circ}$, 远大于平滑聚苯乙烯膜的接触角 $\left(91^{\circ}\right)$. 温度梯度场的 构建是在氮气保护下膜的一端为 $130{ }^{\circ} \mathrm{C}$, 保持 $48 \mathrm{~h}$, 而 膜的另一边则保持室温. 2011 年奥克兰大学 Miskelly 等 ${ }^{[68]}$ 利用坚直扩散梯度法对一维多孔硅光子晶体进行 烯烃的化学氢化硅烷化处理，从而在多孔硅壁表面构建 梯度烯烃分布(图 2a). 为了增加样品的稳定性, 还需要 利用电化学法对样品进行甲基化处理. 由烯烃组成梯度 引起多孔硅表面的梯度浸润性，当表面组成由高浓度的 甲基变为高浓度的 10-羧基癸基或 10-羟基癸基时，相应 的接触角从 $75^{\circ}$ 变为 $60^{\circ} .2012$ 年哈佛大学 Aizenberg ${ }^{[69,70]}$ 提出 “Wetting In Color Kit”, 她通过结合 硅烷基化表面化学和等离子体氧化方法将反蛋白石结 构 $\mathrm{SiO}_{2}$ 光子晶体引入坚直浸润性梯度, 可以用于区分 乙醇浓度、醇类(图 2b)、烷类(己烷、庚烷、辛烷、壬烷 和癸烷)及汽车燃料(汽油和柴油). 2013 年上海交通大学 胡晓斌课题组 ${ }^{[71]}$ 利用模板法制备离子交换反蛋白石光 子晶体膜，并通过离子交换过程实现胶体光子晶体膜的 梯度浸润性(图 2c), 这种光子晶体膜可以区分毫摩量级 的常见表面活性剂(阴离子、两性离子、非离子和阳离 子表面活性剂)、检测 $10^{-5} \mathrm{~mol}$ 量级小分子硫醇和还原 型烟酰胺腺嘌呤二核苷酸磷酸，该方法响应速度快、抗 干扰性强，可以应用于防伪和可视检测等领域. 2016 年 中科院理化技术研究所王京霞等 ${ }^{[72]}$ 利用模板法制备得 到聚离子液体反蛋白石结构光子晶体膜, 该膜随着溶剂 梯度蒸发可发生自驱动过程: 样品从平整变为弯曲, 再 蜷缩为环状 (图 2d). 在整个驱动过程中, 溶剂的梯度去 浸润过程导致膜发生非均一的膨胀和收缩, 这是样品产 生自驱动行为的主要驱动力. 该体系驱动过程的本征驱 动力源于溶剂和高分子之间的强的氢键作用. 这对新型 溶剂驱动材料的设计和制备具有重要意义. 2017 年吉林 大学薛培宏等 ${ }^{[73]}$ 通过倾斜刻蚀与胶体晶体辅助刻蚀相 结合制备出具有梯度浸润性的硅纳米雉阵列. 通过对梯 度硅纳米锥阵列进行亲水或疏水基团修饰实现对水流 动情况的控制. 例如: 当用温敏材料聚异丙基丙烯酰胺 修饰阵列后，可实现温度精确实时控制水流方向. 这使 梯度浸润性材料在微流控芯片的智能开关及浸润材料 
的高通量篎选等方面有潜在的应用价值.

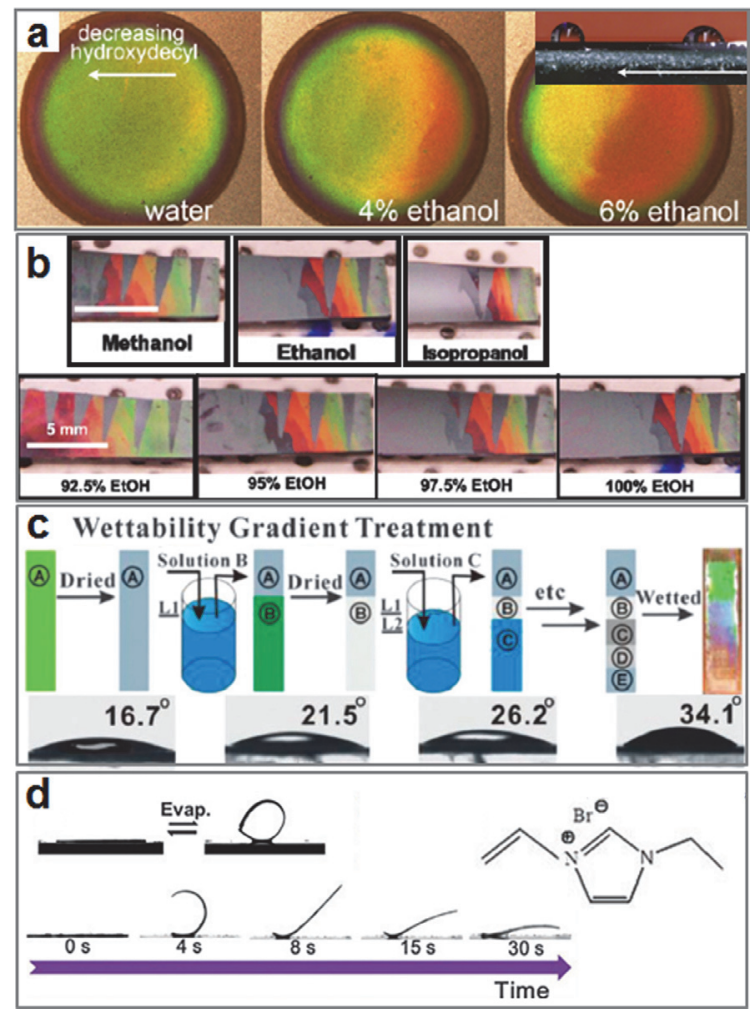

图 2 (a)一维多孔硅光子晶体表面从 10-羟基癸基到甲基的梯度修饰 和乙醇浓度监测 ${ }^{[68]}$; (b) 利用厚度方向具有梯度浸润性的反蛋白石结构 进行可视检测乙醇和水的混合物及醇类 ${ }^{[69]}$; (c) 具有梯度浸润性的反蛋 白石结构水凝胶的两亲分子响应 ${ }^{[71]}$; (d)溶剂响应的聚离子液体反蛋白 石结构梯度去浸润时的驱动行为 ${ }^{[72]}$

Figure 2 (a) The $1 \mathrm{D}$ porous Si photonic crystal modified with a gradient of 10-hydroxydecyl to methyl and ethanol concentration detection ${ }^{[68]}$. (b) Colorimetric distinction of different ethanol-water mixtures and alcohols using an inverse-opal films functionalized with a vertical gradient wettability ${ }^{[69]}$. (c) The inverse opal hydrogel films with wettability gradients and its amphiphilic molecular response ${ }^{[71]}$. (d) The actuating behavior of solvent-sensitive PIL inverse opals based on gradient dewetting ${ }^{[72]}$

\section{5 可调控浸润性胶体光子晶体膜}

\subsection{1 静态浸润性可调控胶体光子晶体膜}

可调控浸润性胶体光子晶体膜是指膜表面的浸润 性对外界刺激(温度、 $\mathrm{pH}$ 、光、电和热)发生变化. 其通 常有两种制备方法: 由亲疏水链段组成的乳胶粒子可以 通过调控温度或 $\mathrm{pH}$ 值来调整其表面组成, 实现对其浸 润性的调控; 另外将对外界刺激响应的分子制备成光子 晶体或引入到光子晶体表面, 同时结合光子晶体的本征 有序粗粘结构, 实现对胶体光子晶体膜浸润性的可逆调 控. 2006 年中科院化学研究所王京霞等 ${ }^{[74]}$ 通过调控组装 温度调控乳胶粒表面的化学组成, 最终实现膜表面浸润 性的调控, 当组装温度从 $20{ }^{\circ} \mathrm{C}$ 到 $90{ }^{\circ} \mathrm{C}$ 变化时所制备 膜的水接触角从 $0^{\circ}$ 到 $150.2^{\circ}$ 变化. 为了满足不同使用温 度对膜浸润性的应用需求, 王京霞等 ${ }^{[75]}$ 又进一步调整 组成乳胶粒的软硬聚合物链段比例, 从而实现 $20 \sim$ $90{ }^{\circ} \mathrm{C}$ 之间均可制备超疏水光子晶体膜. 之后, 她们又 研究了组装液 $\mathrm{pH}$ 值对所组装膜表面浸润性的影响(图
$3 a)^{[76]}$. 胶体光子晶体膜的浸润性还可以通过光 ${ }^{[77 ~ 79] 、}$ 电 ${ }^{[80 ~ 82]}$ 和热 ${ }^{[83,84]}$ 外场可逆调控. 中科院化学研究所葛 红莉等 ${ }^{[77]}$ 利用偶氮分子在光照前后发生顺反异构变化 并结合光子晶体粗糙结构对浸润性的放大效应, 实现膜 接触角由紫外光照前的 $140.8^{\circ} \pm 3.0^{\circ}$ 变化为紫外光照后 的 $121.8^{\circ} \pm 4.3^{\circ}$. 随后, 中科院化学研究所徐亮等 ${ }^{[80}$ 通 过电化学沉积方式制备得到聚吡咯反蛋白石结构光子 晶体. 利用聚吡咯在不同氧化还原态的掺杂与脱掺杂引 起的体积、折光指数及导电性的变化, 制备得到了导电 性、浸润性及光子带隙随不同氧化还原状态可逆调控的 聚合物光子晶体膜(图 3b). 2010 年法国国家科研中心的 Mangeney 等 ${ }^{[83]}$ 制备出热响应胶体光子晶体, 其浸润性 可以随温度变化从亲水到疏水可逆调控. 2017 年, 中科 院理化技术研究所王京霞等 ${ }^{[85]}$ 制备了一类金属-有机反 蛋白石结构光子晶体, 该光子晶体在电浸润过程中浸润 性发生了从疏水性 $\left(120^{\circ}\right)$ 到亲水性 $\left(56.2^{\circ}\right)$ 的变化. 有趣 的是样品的形貌在电浸润过程中也发生了独特的演变 (图 3c): 从典型的贯通反蛋白石结构逐渐演变为分隔独 立的空心粒子组装阵列. 实验结果表明该过程得到的并 不是单一的硝酸铅反蛋白石结构, 还包括由铅离子与来 自于嵌段聚合物羧基形成的鳌合物的复合金属有机反 蛋白石结构. 其中的硝酸铅在电浸润过程中会溶解在水 中, 导致原来结构的坍塌, 而其中的铅离子与羧基形成 的螯合物会在原有结构坉塌的基础上重新组装排列. 最 终形成了分隔独立空心粒子组装体. 作者还尝试利用该 电浸润方法实现光子晶体图案化制备(图 3d), 这为发展 简单、有效、低成本水刻图案制备提供新思路.

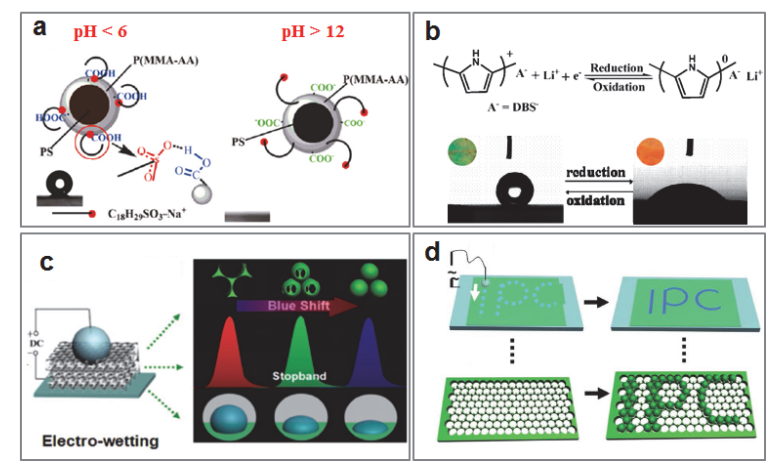

图 3 调控浸润性通过(a) $\mathrm{pH}$ 值 ${ }^{[76]}$ 和(b)电化学氧化还原 ${ }^{[80]}$; (c)电浸润 过程中的形貌、反射光谱和浸润性变化 ${ }^{[85]}$ 和(d)电浸润图案制备 ${ }^{[85]}$ Figure 3 (a) Controlling wettability of PCs by $\mathrm{pH}^{[76]}$. (b) Tunable wettability of PCs with electrochemical redox ${ }^{[80]}$. (c) Manipulating the morphology, reflectance spectrum and wettability through electrowetting and (d) schematic illustrations for pattern formation obtained by the electrowetting process ${ }^{[85]}$

\subsection{2 动态浸润性可调控胶体光子晶体膜}

特殊粘附性胶体光子晶体通常是由各向异性粒子 自组装制备得到 ${ }^{[23,86 ~ 92]}$. 受自然界玫瑰花瓣的特殊微结 构对水的超粘附性能的启发, 中科院化学研究所黄羽等 通过控制乳液聚合过程，实现了对乳胶粒表面形貌和粗 䊁结构的调控，制备了球形、单腔和菜花形状乳胶粒， 
并进一步研究了这些乳胶粒组装成的胶体晶体膜在水 下对油的粘附性能 ${ }^{[23]}$ [图 4(a c ) ], 发现光滑的球形乳胶 粒所组装的光子晶体在水下显示了超强的油粘附性能, 菜花状乳胶粒所组装的表面则显示了较小的油粘附力, 而单腔乳胶粒组装结构在水下显示了非常低的油粘附 力. 不同乳胶粒组装结构所形成的与油滴的三相接触线 由连续到不连续变化从而影响其粘附力. 这种简单制备 特殊粘附性膜的方法对超粘性材料的制备具有重要意 义. 特殊粘附性胶体光子晶体也可以通过将液体注入二 维多孔胶体晶体表面实现, 这种方法是 2013 年哈佛大 学 Aizenberg 课题组 ${ }^{[58]}$ 提出的(图 4d). 具体是以二维胶 体晶体为模板制得 $\mathrm{SiO}_{2}$ 多孔有序结构, 接着除去模板 后再氟硅烷处理, 最后注入润滑油实现抗粘附表面的制 备, 可以阻止各种液体污染物和冰的粘附. 最近, 东南 大学赵远锦课题组 ${ }^{[93]}$ 发展了一种用于自报告的可调控 表面粘附性光子晶体. 他们将(perfluorinated oil)油填充 在所制备的聚氨酯反蛋白石样品中, 油填充后的样品显 示了对油和水滴的良好滑移性能. 值得关注的是, 当将 该样品进一步拉伸时, 拉伸后的样品会使其表面的油或 水滴由滑移态转变为粘附态(图 4e). 在这个过程中, 样 品表面的结构色彩也发生非常明显的变化, 可以作为检 测膜动态浸润行为的一种有效方法.

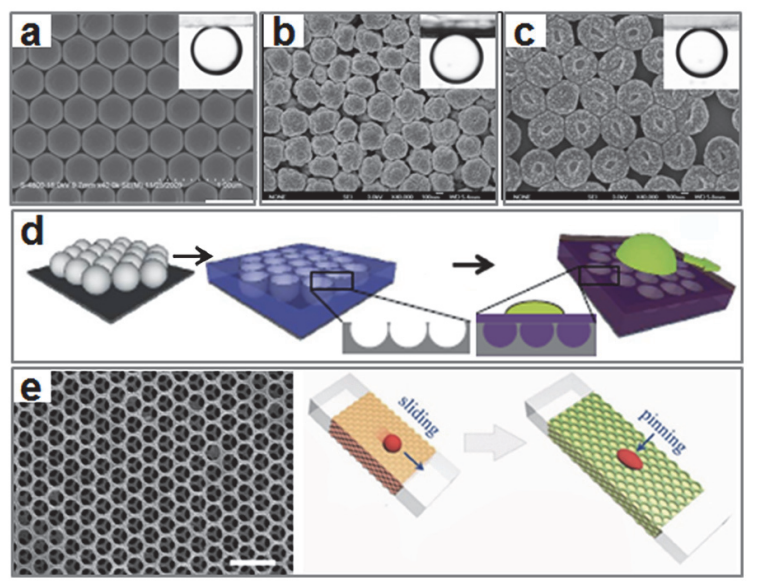

图 $4(\mathrm{a} \sim \mathrm{c})$ 球形、菜花和单腔乳胶粒组装结构的扫描电镜图, 插图是 当膜在水下时油滴在其表面的形状 ${ }^{[23]}$; (d)液体注入多孔膜制备抗粘附 光子晶体 ${ }^{[58]}$; (e) 聚氨酯反蛋白石结构 SEM 图和通过其表面粘附性调 控液滴移动 ${ }^{[93]}$, 标尺: $1 \mu \mathrm{m}$

Figure $4 \quad(\mathrm{a} \sim \mathrm{c})$ SEM images of the films assembled from the latex particles with spherical, cauliflower-like and single-cavity latex structures. The inset images are the shapes of the oil droplets on the films on immersion in water ${ }^{[23]}$. (d) Fabrication of lubricant-infused inverse monolayers ${ }^{[58]}$. (e) SEM image of the PU inverse opal nanostructure and control of droplet mobility on its lubricant-infused surface ${ }^{[93]}$, scale bar: $1 \mu \mathrm{m}$

\section{6 图案浸润性胶体光子晶体}

通过对胶体光子晶体不同区域的浸润性进行设计 从而制备图案浸润性胶体光子晶体. 图案胶体光子有望 应用于集成光路, 但关于图案浸润性胶体光子晶体的研 究相对较少, 文献中关于其制备主要是借助模板法, 并 且需要多步骤实现 ${ }^{[94 ~ 98]}$. 例如: 哈佛大学的 Aizenberg
等 ${ }^{[94]}$ 利用多次烷基化处理和氧等离子体处理法在反蛋 白石 $\mathrm{SiO}_{2}$ 光子晶体膜上实现图案浸润性的构筑(图 5a). 光子晶体的结构色和浸润性图案结合可以用于防伪和 检测. 比利时鲁汶大学的 Clays 和中科院理化技术研究 所宋恺 ${ }^{[95]}$ 合作报道利用氧等离子体刻蚀实现具有超疏 水/超亲水图案浸润性空心球胶体光子晶体膜的制备(图 $5 b)$, 这种膜可以用于实时超灵敏荧光检测. 澳大利亚 弗林德斯大学 Voelcker 等 ${ }^{[97]}$ 通过结合光刻胶图案和表 面烷基化处理在多孔硅表面实现图案浸润性的制备(图 $5 \mathrm{c})$, 并应用于有机气体传感。最近, 韩国科学技术院 Kim 等 ${ }^{[98}$ 通过对反蛋白石结构光子晶体膜进行图案化 氧等离子体刻蚀及聚乙二醇接枝实现柔性、自支撑反蛋 白结构的编码(图 5d), 并且这种结构可以转移到其它表 面、实现重复使用及长期保存.

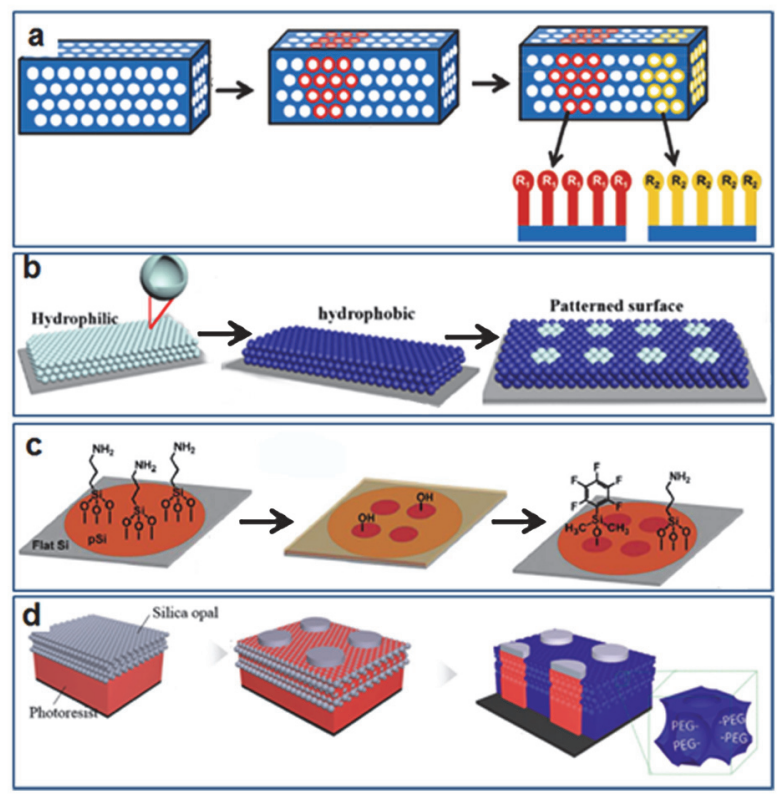

图 5 (a)光子晶体复杂浸润性图案制备示意 ${ }^{\left[{ }^{[}\right.}{ }^{[4]}$; (b)超疏水/超亲水图 案空球胶体光子晶体的制备示意图 ${ }^{[95]}$; (c)两种硅烷偶联剂在多孔硅表 面图案化接枝过程 ${ }^{[97]}$; (d)制备高分子反蛋白石结构区域浸润性编码示 意图 ${ }^{[98]}$

Figure 5 (a) Schematic of complex wettability patterns fabrication in 3D photonic crystals ${ }^{[94]}$. (b) Schematic illustration of the procedure for introducing superhydrophobic/superhydrophilic patterns into $\mathrm{HSCPCs}^{[95]}$. (c) Surface fabrication procedure for the patterned incorporation of two different silane compounds on a $\mathrm{pSi}$ (porous silicon) rugate surface ${ }^{[97]}$. (d) Schematic illustration of encryption procedures to create polymeric inverse opals of which pore wettability is regioselectively modified ${ }^{[98]}$

\section{3 基底浸润性对组装胶体光子晶体功能性的影 响}

光子晶体的特殊浸润性可以影响溶液在光子晶体 表面的浸润铺展行为, 影响其最终功能性及其相关应 用. 组装基底的浸润性可以有效调控胶乳在基底的浸 润、铺展及蒸发过程, 影响胶乳蒸发过程中的组装作用 力、乳胶粒的组装行为及最终组装结构, 从而调控所组 装光子晶体的功能性及相应应用. 对于传统的胶体晶体 
组装, 亲水、超亲水基底 ${ }^{[99 \sim 101]}$ 有利于组装液在其上的充 分均匀铺展, 得到连续均质的光子晶体 ${ }^{[17,19,22]}$. 早期的 胶体晶体组装结构主要通过亲水、超亲水基底制备得到. 近年来, 随着浸润性研究的深入开展, 静态浸润性, 动 态浸润性(包括低粘附、高粘附及滚动角等)对胶乳蒸发 过程及组装过程的影响得到广泛关注, 更多特殊浸润性 基底的组装光子晶体的研究工作也得到开展, 拓展了光 子晶体的很多功能及应用.

\section{1 疏水基底}

光子晶体由于其特殊的光操控性能在集成光学器 件, 全色显示和生物传感等领域广泛应用. 但通常制备 的光子晶体膜的结构色具有角度依赖性，限制了其在广 角显示领域的应用. 疏水基底为实现优秀广角性半球光 子晶体制备提供了有效途径 ${ }^{[102 ~ 109]}$. 2008 年韩国科学技 术院 Yang 教授课题组 ${ }^{[102]}$ 利用二氧化硅微球结合乙氧基 三羟甲基丙烷三丙烯酸酯光固化树脂在聚四氟乙烯涂 覆的玻璃基底上制备红、绿、蓝三色半球图案 [图 6(a,b)]. 两面(图 6c)或三面半球图案可以通过两个或三个液滴融 合制备, 这种半球图案也可以在柔性 PDMS 表面实现, 并且还可以通过湿刻蚀除去二氧化硅微球来提高反射 强度. 2014 年中科院化学研究所广旻噮㩐 ${ }^{[103]}$ 通过调控 液滴在疏水基底上的三相线移动并结合喷墨打印技术 实现了具有光子晶体半球结构图案的制备(图 6d). 这些 半球光子晶体为有效解决结构色角度依赖性提供有效 途径. 同一实验室的刘美金等 ${ }^{[104]}$ 通过墨滴融合时的动 态浸润性在疏水基底上实现了连续光子晶体线的制备 (图 6e). 这个研究将推动打印光子晶体连续电子器件的 发展. 之后, 同实验室鲍斌等 ${ }^{[105]}$ 利用反应型喷墨打印 制备量子点纳米复合物. 其中禁带匹配的光子晶体的引 入可以增强量子点的荧光发射. 这种简单的原位打印制 备方法可广泛应用于各种纳米复合材料图案的制备. 最 近，日本千叶大学的 Kohri 等 ${ }^{[106]}$ 通过混合两种不同尺寸 的核壳粒子实现全波段结构色调控(图 6f), 其中聚苯乙 烯为核, 聚多巴胺为壳, 光子晶体结构色墨水的应用和 发展对于其在颜料方面的应用具有非常重要的意义.

\section{2 超疏水基底}

光子晶体具有特殊的周期性结构, 能够调控光的传 播和发射, 又被称为 “光学半导体”, 被认为是未来光 子工业的材料基础, 其研究和应用受到广泛关注. 2008 年北卡罗莱纳州大学的 Velev 等 ${ }^{[110]}$ 在低密度聚乙烯或 硅纳米线超疏水基底上制备了球形胶体组装结构(图 $7 \mathrm{a})$, 进一步引入金属粒子可以提高衍射颜色. 所制备结 构可以应用于药物输运、光子、涂层、传感和微流体等 领域. 2010 年北卡罗莱纳州大学的 Velev 等 ${ }^{[111]}$ 在超疏水 基底利用液滴模板法实现了甜甜圈状和各向异性结构 (patchy)超粒子的制备(图 7a). “甜甜圈” 组装采用的是 $\mathrm{SiO}_{2}$ 微球或其与金纳米粒子混合得到, 各向异性结构采

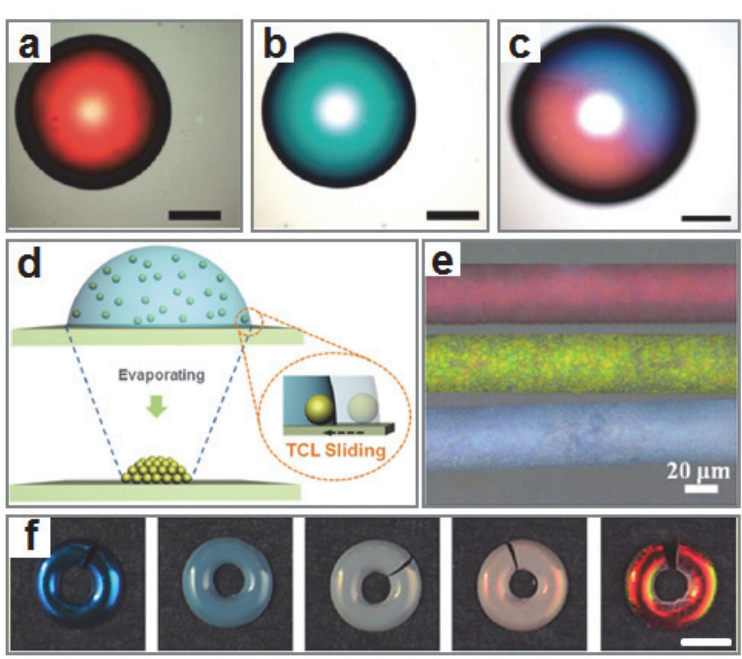

图 $6 \quad(a \sim c)$ 在聚四氟乙烯涂覆的玻璃表面制备红、绿和两面半球光 子晶体图案 ${ }^{[102]}$, 标尺: $100 \mu \mathrm{m}$; (d)喷墨打印制备拱形光子晶体结构的 组装示意图 ${ }^{[103]} ;$ (e) 喷墨打印制备的光子晶体红、绿、蓝线结构 ${ }^{[104]}$; (f) 通过混合胶体粒子对结构色进行调控 ${ }^{[106]}$, 标尺: $5 \mathrm{~mm}$

Figure $6 \quad(\mathrm{a} \sim \mathrm{c})$ The Red, green and janus hemispheres on a Teflon-coated glass substrate ${ }^{[102]}$. Scale bar is $100 \mu \mathrm{m}$. (d) Illustration for the assembling process of PC domes on an OTS-treated substrate ${ }^{[103]}$. (e) Three typical straight PC lines (red, green, and blue) fabricated by inkjet printing $^{[104]}$. (f) Structural colors manipulation by mixing colloidal particles ${ }^{[106]}$, scale bar: $5 \mathrm{~mm}$

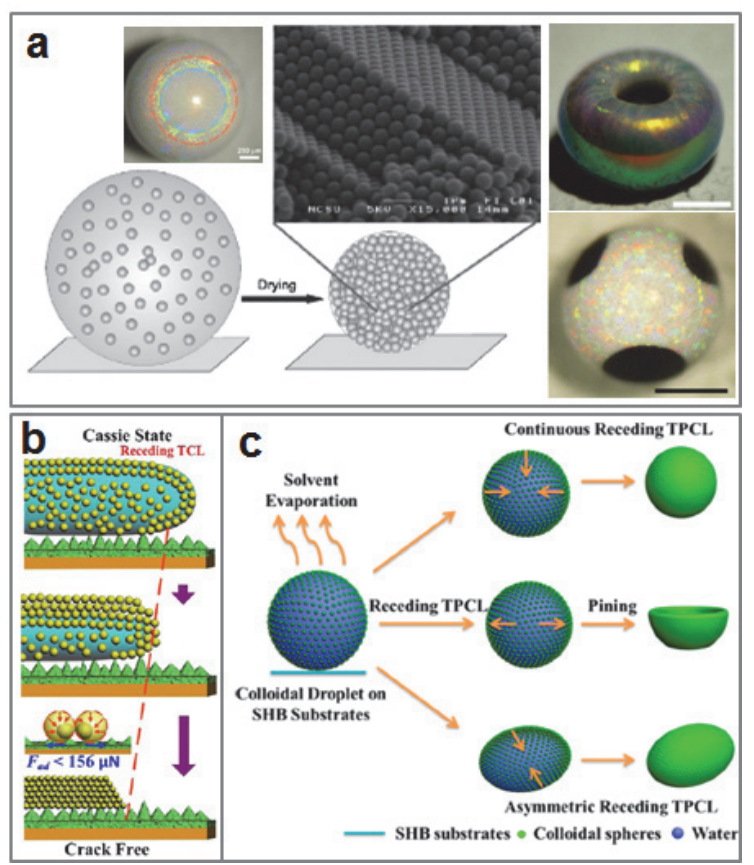

图 7 (a)在超疏水基底制备球形、甜甜圈和补丁形胶体光子晶体组装 结构 ${ }^{[110,111]}$, 标尺: $500 \mu \mathrm{m}$; (b)在超疏水低粘附基底上制备无裂纹胶体 光子晶体 ${ }^{[113]}$; (c) 在超疏水基底通过调控三相线调控光子晶体组装结 构 ${ }^{[14]}$

Figure 7 (a) Spherical, doughnut and patchy colloidal assembly on superhydrophobic surface ${ }^{[110,111]}$. Scale bar: $500 \mu \mathrm{m}$. (b) Large-scale crack-free colloidal PCs are achieved on low Fad superhydrophobic substrate $^{[113]}$. (c) Schematic illustrations of the microshape controllable fabrication of PC assemblies by facile evaporation of colloidal droplets on SHB substrates based on different dynamic behaviors of the TPCL ${ }^{[114]}$

用的是聚苯乙烯微球和磁粒子混合, 粒子聚集图案可由 磁场调控. “甜甜圈” 超粒子可应用于催化, 而各向异 
性超粒子组装可应用于药物输运和微流体领域. 2012 年 特温特大学的 Marín 课题组 ${ }^{[112]}$ 也在超疏水微结构基底 实现胶体微结构的构筑, 并通过粒子数量和聚集率对微 结构进行调控, 在整个蒸发过程中液滴始终处于 Cassie-Baxter 态. 微结构的尺寸可以通过湿度、温度和 聚集率调控, 而且这种微结构可以通过喷涂方法大量制 备得到 ${ }^{[112]}$.

2012 年中科院化学研究所黄羽等 ${ }^{[113]}$ 在超疏水低粘 附基底上实现厘米尺寸、窄带隙、高质量胶体光子晶体 的制备, 其半峰宽仅为 $12 \mathrm{~nm}$. 窄的半峰宽主要是源于 乳胶球的有序组装、所制备膜的高质量(大面积无裂缝), 及降低的空隙率和足够的厚度(图 7b). 该文还研究了基 底粘附力对胶体光子晶体组装结构的影响, 仅当基底粘 附力小于 $60 \mu \mathrm{N}$ 时能实现半峰宽为 $12 \mathrm{~nm}$ 胶体光子晶体 的制备. 这种高质量光子晶体的制备方法对高级光学器 件的设计和制备具有重要意义. 2015 年河北师范大学周 金明等 ${ }^{[114]}$ 又在超疏水基底上通过调控三相接触线实现 了微球、非对称微孔和微椭球形状胶体光子晶体的制 备. 所使用基底的接触角为 $151^{\circ}$, 形貌表征显示所制备 的结构无裂纹. 表层乳胶球进行了有序组装, 而内部乳 胶球是无规则排列. 他们进一步将黑色 $\mathrm{Fe}_{3} \mathrm{O}_{4}$ 引入到该 体系中, 所制备的胶体晶体结构不仅具有超顺磁性能, 而且还具有更加明显的广角非彩虹结构色. 不同形貌组 装结构的形成机理主要是源于可控的气液固三相接触 线(图 7c): 当胶体液滴在超疏水基底上蒸发时, 其三相 线发生均一连续收缩, 则有助于形成微球状胶体光子晶 体; 当其三相线锁定, 这种情况下液滴蒸发时则有助于 形成带酒窝微球或微孔状光子晶体; 当三相线非对称性 后退即在一个方向锁定, 在与其垂直方向均一收缩, 这 种情况则有助于形成各向异性胶体自组装. 这种制备胶 体晶体微结构的方法对制备新型胶体粒子微结构具有 重要的意义.

\section{3 亲-疏水图案化基底}

光子晶体在光电器件等领域具有巨大的应用前景, 因而乳胶球的精确组装与图案化制备成为光子晶体研 究领域的一个热点. 亲-疏水图案基底的设计对于实现 图案化光子晶体的精确制备非常有意义. 2002 年, 东南 大学顾忠泽等 ${ }^{[115]}$ 利用亲疏水图案基底制备得到了具有 鱼型的图案化光子晶体(图 8a). 2011 年吉林大学杨柏 等 ${ }^{[116]}$ 基于不同表面能图案制备得到具有国际象棋形状 的图案化光子晶体(图 8b). 2013 年中科院化学所宋延林 课题组 ${ }^{[117]}$ 在亲-疏水图案基底上制备了光子晶体微芯 片, 利用 8-差基喹啉可以实现对 12 种金属离子 $\left(\mathrm{Al}^{3+}\right.$, $\mathrm{Ca}^{2+}, \mathrm{Cd}^{2+}, \mathrm{Co}^{2+}, \mathrm{Cr}^{3+}, \mathrm{Cu}^{2+}, \mathrm{Fe}^{2+}, \mathrm{Hg}^{2+}, \mathrm{Li}^{+}, \mathrm{Mg}^{2+}, \mathrm{Ni}^{2+}$ 和 $\mathrm{Zn}^{2+}$ ) 的识别和分析. 这种多带隙光子晶体微芯片对 于高效多元分析方法的发展具有重要意义. 2015 年该课 题组 ${ }^{[118]}$ 提出一种基于亲疏水图案制备三维光子晶体微 结构的简便方法(图 8c). 通过在疏水基底引入可控零维
亲水图案，调控液滴的固-液-气三相线使液滴发生非对 称去浸润，从而制得三角形、四边形、五边形、六边形 和八边形胶体晶体微图案. 该方法操作简便, 具有广泛 的普适性. 这一研究成果为快速制备形貌可控的精细 3D 结构提供了新的思路, 对 3D 打印技术的发展具有重 要意义和启示. 2016 年中科院理化技术研究所王京霞 等 ${ }^{[119,120]}$ 将单分散胶乳液填充在超亲水基底与超疏水硅 柱形成的三明治结构中, 利用疏水硅柱的限域作用, 实 现了光子晶体线、微环(图 8d)、方形等微阵列的制备, 并 研究了所制备光子晶体微环的光波导性能(图 8e). 2017 年乔治亚理工学院林志群课题组 ${ }^{[121]}$ 在亲疏水图案基底 上得到胶体自组装的大面积周期裂缝, 并利用裂缝作为 模板引导 $\mathrm{Au}$ 纳米粒子组装.
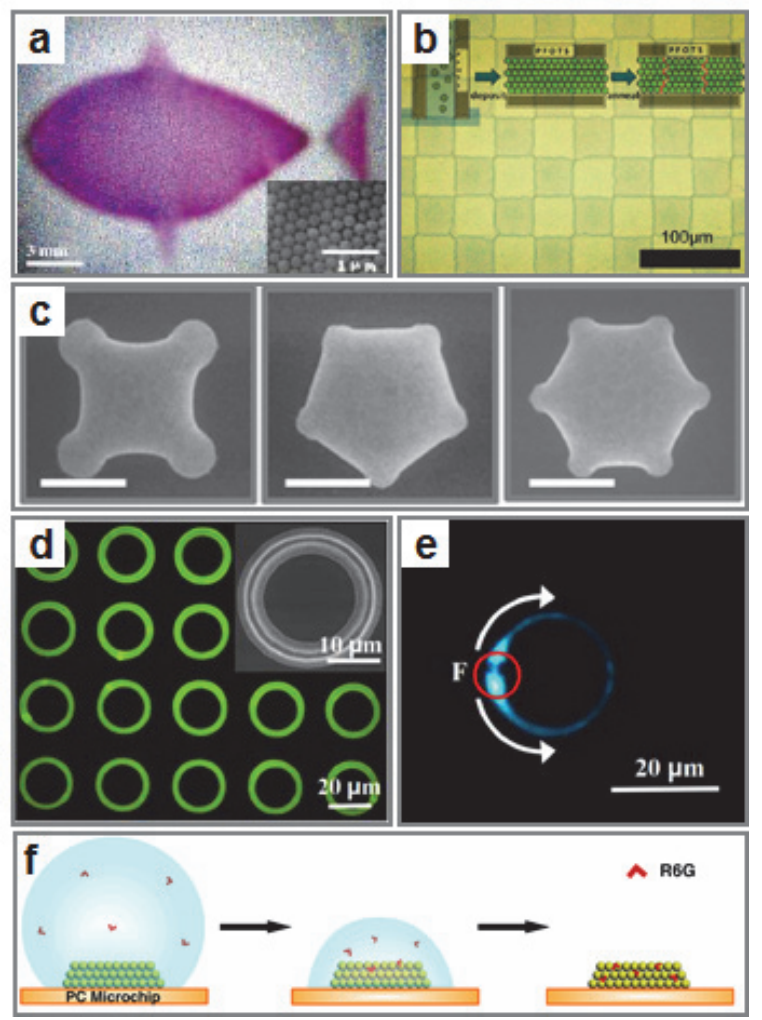

图 8 图案浸润性基底上制备的(a)鱼 ${ }^{[115]}$ 、(b)国际象棋 ${ }^{[116]}$ 、(c)四边形、 五边形、六边形图案光子晶体 ${ }^{[118]}$, 标尺: $20 \mu \mathrm{m}$; (d)环形胶体光子晶体 (e)它的波导性能 ${ }^{[119]}$; (f) 亲疏水光子晶体图案诱导苂光分子富集示意 图 ${ }^{[123]}$

Figure 8 (a) The fish-shaped ${ }^{[115]}$ and (b) international chess pattern fabricated on pattern wettability substrates ${ }^{[16]}$. (c) The controllable 3D structure include quadrilateral, pentagon and hexagonal from left to right through hydrophilic pattern induced asymmetric dewetting ${ }^{[118]}$. Scale bar: $20 \mu \mathrm{m}$. (d) Microring pattern and (e) its waveguide property ${ }^{[119]}$. (f) The scheme for the enrichment process of the fluorescence molecules (R6G) by hydrophilic-hydrophobic micro-pattern ${ }^{[123]}$.

受沙漠甲虫(Stenocara beetle)背部具有收集雾滴能 力的亲/疏水图案的启发, 将光子晶体苂光增强特性与 表面浸润性图案(亲水光子晶体-疏水基底)相结合, 将 其先后应用于无标记蛋白检测 ${ }^{[122]}$ 、苂光痕量检测 ${ }^{[123]}$ 和 基于分子印迹的四环素检测 ${ }^{[124]}$. 2012 年中科院化学研 
究所沈为之等 ${ }^{[122]}$ 在疏水基底上打印制备 $\mathrm{Y}$ 型光子晶体 微流体芯片. 基底的接触角为 $125.8^{\circ} \pm 2.1^{\circ}$, 光子晶体 通道的接触角为 $57.8^{\circ} \pm 2.5^{\circ}$. 结合基底的疏水性和光子 晶体的亲水性, 该浸润性图案可应用于无标记蛋白检 测. 这种芯片制备过程简单快速, 使用时易于操作, 可 以实时监控流体传输, 避免非特异性吸附干扰, 具有高 效稳定性. 随后, 同实验室的侯觉等 ${ }^{[123]}$ 利用喷墨打印 技术在疏水基底上制备了亲水性光子晶体阵列, 利用其 表面浸润性差异将水滴中的待检测物富集到检测位点亲水性光子晶体点阵(图 8f), 同时利用光子晶体来增强 荧光信号, 从而实现了对荧光分子和基于荧光方法的分 子检测系统的高灵敏分析检测. 该方法简便易行, 适用 范围广, 能够实现大面积高通量的检测阵列制备, 用于 发展高灵敏、高通量的光学检测器件. 之后, 该作者又 将亲疏水图案基底的富集效应应用于光子晶体分子印 迹检测器中, 构筑具有亲疏水图案的光子晶体分子印迹 色度传感器, 基于蒸发富集原理提升光子晶体区域中的 待检测物浓度，从而提升检测灵敏度 ${ }^{[124]}$.

\section{4 液体表界面合成/组装光子晶体}

液体表面、气液界面或液-液界面为不同表面能基 材提供更宽广的舞台, 这些界面已经发展成为一种有效 的方法实现特殊形貌粒子的制备及特殊功能粒子的组 装. 2002 年, 德国美因茨大学 Zentel 等 ${ }^{[125]}$ 采用溶解的液 体 $\mathrm{Ga}$ 制备大面积无裂纹光子晶体, 可以有效避免由于 固体基材对粒子组装过程中收缩的限制. 基于类似的思 想, 2012 年, 美国匹兹堡大学 Asher 等 ${ }^{[126]}$ 采用液体 $\mathrm{Hg}$ 基材制备大面积高质量的光子晶体(图 9a). 气一液界面 已经被发展为一种有效的方法实现各向异性粒子的制 备. 2010 年, 中科院化学所徐亮等 ${ }^{[89]}$ 合成了一种具有蘑 菇头形状的乳胶粒, 该粒子凸起的一面因富含羧基更为 亲水, 凹下部分因羧基减少更为疏水, 基于蘑菇头形状 粒子两端的不对称浸润性差异, 可以在空气一水界面进 行有序的组装, 实现了蘑菇头粒子组装的各向异性光子 晶体的大面积制备. 北京大学齐利民课题组 ${ }^{[127 ~ 129]}$ 利用 气液界面胶体球刻蚀法实现了包括多种无机物纳米网、 纳米碗阵列(图 9b)和纳米网-纳米碗复合阵列在内的一 系列自支撑二维有序多孔薄膜的可控制备, 考察了其二 维光子晶体性质, 并研究了其在刻蚀掩膜、溶剂检测、 生物传感、电阻开关器件、光电化学分解水等方面的应 用. 在上述体系中, 空气一水界面为软且不对称的规整 结构制备提供了一个多样的平台.

最近, 中科院理化技术研究所王京霞的系列研究工 作 ${ }^{[130 ~ 133]}$ 推进了气-液或液-液界面的粒子制备及组装. 2015 年, 该课题组通过调控(固体)界面浸润性, 借助水 滴模板法实现对所制备粒子形状(环、饼、烧瓶)的调 控 ${ }^{[130,131]}$. 具体的实验过程包括首先在固体基材上形成 水滴模板, 然后将功能分子滴涂在水滴模板上面, 功能 分子会包覆在水滴模板表面, 形成特定形状的粒子. 在

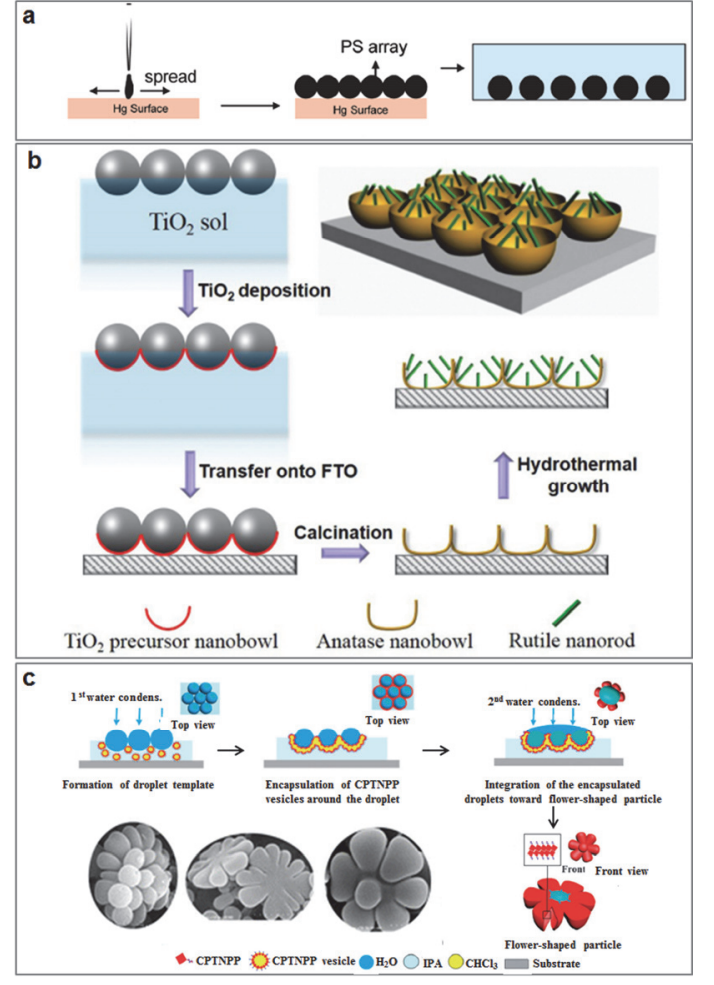

图 9 (a)在 $\mathrm{Hg}$ 表面制备二维光子晶体 ${ }^{[126]}$; (b)阵列 $\mathrm{TiO}_{2}$ 纳米碗中生长 纳米棒示意图 ${ }^{[128]}$; (c)两步水滴模板法制备的花形胶体晶体示意图. 先 是在 CPTNPP 溶液表面形成液滴模板, 然后是功能分子包覆液滴组 装, 第二次液滴冷凝伴随粒子的包覆导致花形粒子的形成. 插图是花 形粒子的扫描电镜图 ${ }^{[133]}$

Figure 9 (a) Fabrication of a 2D photonic crystal on mercury surface ${ }^{[126]}$. (b) Schematic illustration of the preparation process of $\mathrm{TiO}_{2}$ NR@NB arrays ${ }^{[128]}$. (c) Scheme for the formation of the flower-shaped colloidal crystals by the second-step water droplet template. Formation of the droplet template upon CPTNPP suspension, and the encapsulation of the template by suspension accompanied with the droplet assembly, 2nd droplet condensation integration part of the encapsulated particles toward the flower-shaped particles. The insets are the SEM images for the formation of flower-shaped particles ${ }^{[133]}$

这个过程中, 功能分子会在水滴模板与功能溶液的界面 聚集，所形成粒子的形状由水滴模板在溶液中的形状决 定. 根据文中推导的液-固-液界面的改性的 Young 氏方 程, 粒子的形状取决于水滴与功能溶液的表面张力及相 互间的界面张力. 有意思的是, 在界面组装的过程不仅 实现了特定形状的粒子的制备，其中的功能分子也实现 了有序组装, 且呈现了单晶性能. 随后, 该课题组进一 步报道了液一液界面不仅实现各向异性粒子的制备，还 能同时实现不对称粒子的合成及组装 ${ }^{[132,133]}$. 在他们工 作中, 将含卟啉的功能分子溶液滴涂在基底上, 由于溶 剂挥发引起水滴在功能溶液表面冷凝, 功能分子会在水 滴及功能溶液的界面进行聚集组装, 靠近溶液的水滴侧 功能分子组装比较密实, 保持水滴凸起的球形, 而朝向 空气侧的水滴表面会有很少的功能分子包覆聚集，因此 最终会形成类似面包的一端凸起(图 9c), 一端平的各向 异性粒子的制备 ${ }^{[132]}$. 值得借鉴的是，由于水滴冷凝的 过程实现了有序的排列及组装，导致形成的各向异性粒 
子也实现了有序的组装. 最终引起各向异性粒子的形成 /组装同步进行. 当进一步控制第二次水滴冷凝，会得到 更为复杂的各向异性粒子, 比如花形粒子的同步合成及 组装 ${ }^{[133]}$ (图 9c). 第二次形成的水滴使前期形成的面包 型粒子进一步堆积成束, 形成了类似花蒂的结构, 而相 反的一侧依然保持粒子饱满的凸起状, 类似绽放的花 瓣. 由于面包型粒子的取向性排列, 所形成的花瓣型粒 子也呈现了非常有序的取向性结构. 这些各向异性的光 子晶体展现了特殊的光学性能. 液-液复合界面为复杂 形状粒子的合成及组装提供了新途径, 为功能性新材料 的制备提供了新思路.

\section{4 展望}

具有特殊浸润性胶体光子晶体膜的制备及功能性 已经受到越来越多科学工作者的关注, 包括超亲液、超 疏液、梯度浸润性、两亲性、可调控浸润性和水下可控 油粘附性胶体光子晶体膜. 尽管胶体光子晶体的浸润性 研究已取得一定进展, 但更多的特殊浸润性光子晶体的 应用研究还需要深入开展. 其中, 关于梯度浸润性胶体 光子晶体膜的研究比较少, 由于其在储能、电催化和热 电领域的潜在应用前景, 其制备和应用都有待进一步研 究. 近年来, 从界面浸润性角度研究胶体光子晶体的组 装结构是一个值得关注的新领域, 包括疏水、超疏水和 图案浸润性基底, 并且将其应用于苂光检测体系中. 但 胶体光子晶体本身仍然有许多热点和难点问题有待进 一步探索和解决. 例如: 形成单分散乳胶粒的物质种 类、功能需要进一步多样化; 可控尺寸、缺陷的高质量 胶体光子晶体的制备; 具有复杂或可控结构的胶体光子 晶体的构筑. 这些问题的解决将推动胶体光子晶体在光 电器件领域的应用具有重要意义.

\section{作者简介}

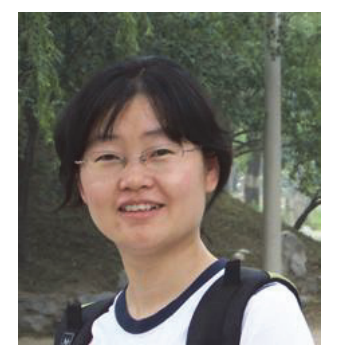

崔丽影, 女, 吉林农业大学实验师, 硕士生导师. 2009 年 在中国科学院化学研究所获得博士学位, 师从宋延林研究员, 研究方向为聚合物光子晶体的简单、大面积制备. 2009 2012 年在吉林大学超分子结构与材料国家重点实验室杨柏教授课 题组做博士后研究, 研究方向为基于微纳有序结构和高分子 添加剂解决咖啡环问题. 2013 年至今, 在吉林农业大学从事教 学科研工作. 目前在包括 Chem. Commun., ACS Appl. Mater. Inter, Small 等国际期刊发表研究论文 30 余篇.

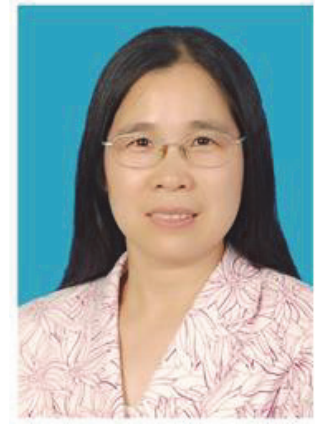

王京霞, 女, 中国科学院理化技术研究所研究员, 博士生 导师，中国科学院大学未来技术学院岗位教授. 多年来聚焦 在浸润性对光子晶体制备及应用性能研究. 2004 年 1 月毕业于 清华大学高分子研究所, 师从刘德山教授. 2004 年 2 月 2006 年 8 月在中科院化学所有机固体实验室江雷研究员课题组做 博士后研究, 研究方向为聚合物光子晶体浸润性研究. 2006 年 8 月 2014 年 4 月留在中科院化学所新材料实验室宋延林研 究员课题组任副研究员, 研究方向为聚合物光子晶体的大面 积制备及应用. 2014 年至今, 调到中科院理化所仿生材料与界 面科学院重点实验室任研究员, 研究方向为特殊浸润性在光 子晶体制备及应用中的作用. 目前在包括 Chem. Soc. Rev., Acc Chem. Res., J. Am. Chem. Soc., Adv. Funct. Mater. 等国际期刊发 表研究论文 90 余篇, 相关研究成果申请并授权专利 40 余项. 参与宋延林研究员主持的项目 “纳米材料绿色打印印刷基础 研究”, 获得 2016 年北京市科学技术一等奖(第四排名)(No. 2016 基-1-001-04).

\section{致谢}

作者衷心感谢中国科学院化学研究所宋延林研究 员和中国科学院理化技术研究所江雷研究员两位导师 一直以来给予的无私指导, 支持和帮助，一并感谢他们 在本文撰写过程中给予的有益讨论和宝贵意见.

\section{References}

[1] Young, T. Philos. Trans. R. Soc. London 1805, 95, 65.

[2] Wenzel, R. N. Ind. Eng. Chem. 1936, 28, 988.

[3] Wenzel, R. N. J. Phys. Colloid Chem. 1949, 53, 1466.

[4] Cassie, A. B. D.; Baxter, S. Trans. Faraday Soc. 1944, 40, 546.

[5] Feng, L.; Li, S. H.; Li, Y. S.; Li, H. J.; Zhang, L. J.; Zhai, J.; Song, Y. L.; Liu, B. Q.; Jiang, L.; Zhu, D. B. Adv. Mater. 2002, 14, 1857.

[6] Zheng, Y. M.; Bai, H.; Huang, Z. B.; Tian, X. L.; Nie, F. Q.; Zhao, Y.; Zhai, J.; Jiang, L. Nature 2010, 463, 640.

[7] Yao, X.; Song, Y. L.; Jiang, L. Adv. Mater. 2011, 23, 719.

[8] Li, K.; Ju, J.; Xue, Z. X.; Ma, J.; Feng, L.; Gao, S.; Jiang, L. Nat. Commun. 2013, 4, 2276.

[9] Tian, Y.; Su, B.; Jiang, L. Adv. Mater. 2014, 26, 6872.

[10] Wen, L. P.; Tian, Y.; Jiang, L. Angew. Chem., Int. Ed. 2015, 54, 3387.

[11] Wang, S. T.; Liu, K. S.; Yao, X.; Jiang, L. Chem. Rev. 2015, 115, 8230 .

[12] Su, B.; Tian, Y.; Jiang, L. J. Am. Chem. Soc. 2016, $138,1727$.

[13] Zhang, P. C.; Lin, L.; Zang, D. M.; Guo, X. L.; Liu, M. J. Small 2017, 13, 1503334 .

[14] Chang, B. S.; Zhang, B.; Sun, T. L. Small 2017, 13, 1503472.

[15] Liu, M. J.; Wang, S. T.; Jiang, L. Nat. Rev. Mater. 2017, 2, 17036.

[16] Sato, O.; Kubo, S.; Gu, Z. Z. Acc. Chem. Res. 2009, 42, 1.

[17] Wang, J. X.; Zhang, Y. Z.; Zhao, T. Y.; Song, Y. L.; Jiang, L. Sci. China Chem. 2010, 53, 318.

[18] Zhang, Y. Z.; Li, Z. R.; Zheng, Y. M.; Wang, J. X.; Song, Y. L.; Jiang, L. Acta Polym. Sin. 2010, 11, 1253. (张佑专, 李志荣, 郑咏 
梅，王京霞，宋延林，江雷，高分子学报, 2010, 11, 1253)

[19] Wang, J. X.; Zhang, Y. Z.; Wang, S. T.; Song, Y. L.; Jiang, L. Acc. Chem. Res. 2011, 44, 405.

[20] Shang, L. R.; Gu, Z. Z.; Zhao, Y. J. Mater. Today 2016, 19, 420.

[21] Wan, L.; Zhang, M. B.; Wang, J. X.; Jiang, L. Acta Chim. Sinica 2016, 74, 639. (万伦, 张漫波, 王京霞, 江雷, 化学学报, 2016, 74, 639.)

[22] Kuang, M. X.; Wang, J. X.; Jiang, L. Chem. Soc. Rev. 2016, 45, 6833.

[23] Huang, Y.; Liu, M. J.; Wang, J. X.; Zhou, J. M.; Wang, L. B.; Song, Y. L.; Jiang, L. Adv. Funct. Mater. 2011, 21, 4436.

[24] Snow, M.; Pring, A.; Self, P.; Losic, D.; Shapter, J. Am. Mineral. 2004, 89, 1353.

[25] Liu, F.; Dong, B. Q.; Liu, X. H.; Zheng, Y. M.; Zi, J. Opt. Express 2009, 17, 16183

[26] Gu, Z. Z.; Fujishima, A.; Sato, O. Appl. Phys. Lett. 2004, 85, 5067.

[27] Li, Y.; Koshizaki, N.; Wang, H. Q.; Shimizu, Y. ACS Nano 2011, 5, 9403.

[28] Tian, E. T.; Ma, Y.; Cui, L. Y.; Wang, J. X.; Song, Y. L.; Jiang, L. Macromol. Rapid Commun. 2009, 30, 1719.

[29] Tian, E. T.; Wang, J. X.; Zheng, Y. M.; Song, Y. L.; Jiang, L.; Zhu, D. B. J. Mater. Chem. 2008, 18, 1116.

[30] Yin, S. N.; Wang, C. F.; Liu, S. S.; Chen, S. J. Mater. Chem. C 2013, 1, 4685.

[31] Xuan, R. Y.; Wu, Q. S.; Yin, Y. D.; Ge, J. P. J. Mater. Chem. 2011, 21,3672 .

[32] Huang, J.; Tao, C. A.; An, Q.; Zhang, W. X.; Wu, Y. G.; Li, X. S.; Shen, D. Z.; Li, G. T. Chem. Commun. 2010, 46, 967.

[33] Huang, J.; Tao, C. A.; An, Q.; Lin, C. X.; Li, X. S; Xu, D.; Wu, Y. G.; Li, X. G.; Shen, D. Z.; Li, G. T. Chem. Commun. 2010, 46, 4103.

[34] Wang, L. B.; Wang, J. X.; Huang, Y.; Liu, M. J.; Kuang, M. X.; Li, Y. F.; Jiang, L.; Song, Y. L. J. Mater. Chem. 2012, 22, 21405.

[35] Li, H. L.; Wang, J. X.; Yang, L. M.; Song, Y. L. Adv. Funct. Mater. 2008, 18,3258 .

[36] Li, H. L.; Chang, L. X.; Wang, J. X.; Yang, L. M.; Song, Y. L. J. Mater. Chem. 2008, 18, 5098.

[37] Gu, Z. Z.; Uetsuka, H.; Takahashi, K.; Nakajima, R.; Onishi, H.; Fujishima, A.; Sato, O. Angew. Chem., Int. Ed. 2003, 42, 894.

[38] Shiu, J. Y.; Kuo, C. W.; Chen, P. L.; Mou, C. Y. Chem. Mater. 2004, 16,561 .

[39] Zhang, X. M.; Zhang, J. H.; Ren, Z. Y.; Li, X.; Zhang, X.; Zhu, D. F.; Wang, T. Q.; Tian, T.; Yang, B. Langmuir 2009, 25, 7375.

[40] Zhang, G.; Wang, D. Y.; Gu, Z. Z.; Möhwald, H. Langmuir 2005, 21, 9143.

[41] Xiu, Y. H.; Zhu, L. B.; Hess, D. W.; Wong, C. P. Langmuir 2006, 22, 9676.

[42] Li, Y.; Li, C. C.; Cho, S. O.; Duan, G. T.; Cai, W. P. Langmuir 2007, 23, 9802 .

[43] Li, Y.; Huang, X. J.; Heo, S. H.; Li, C. C.; Choi, Y. K.; Cai, W. P.; Cho, S. O. Langmuir 2007, 23, 2169

[44] Yao, T. J.; Wang, C. X.; Lin, Q.; Li, X.; Chen, X. L.; Wu, J.; Zhang, J. H.; Yu, K.; Yang, B. Nanotechnology 2009, 20, 065304.

[45] Gao, X. F.; Yan, X.; Yao, X.; Xu, L.; Zhang, K.; Zhang, J. H.; Yang, B.; Jiang, L. Adv. Mater. 2007, 19, 2213.

[46] Ge, H. L.; Song, Y. L.; Jiang, L.; Zhu, D. B. Thin Solid Films 2006, $515,1539$.

[47] Zheng, Y. M.; Gao, X. F.; Jiang, L. Soft Matter 2007, 3, 178.

[48] Yang, H. T.; Jiang, P. Langmuir 2010, 26, 12598.

[49] Raza, M. A.; Kooij, E. S.; Silfhout, A. van; Poelsema, B. Langmuir 2010, 26, 12962.

[50] Park, S. G.; Moon, J. H.; Jeon, H. C.; Yang, S. M. Soft Matter 2012, $8,4567$.

[51] Li, J.; Liang, G. Q.; Zhu, X. L.; Yang, S. Adv. Funct. Mater. 2012, 22, 2980.

[52] Abid, M. I.; Wang, L.; Chen, Q. D.; Wang, X. W.; Juodkazis, S.; Sun, H. B. Laser Photonics Rev. 2017, 1600187.

[53] Tuteja, A.; Choi, W.; Ma, M.; Mabry, J. M.; Mazzella, S. A.; Rutledge, G. C.; Mckinley, G. H.; Cohen, R. E. Science 2007, 318, 1618.

[54] Tuteja, A.; Choi, W.; Mabry, J. M.; Mckinley, G. H.; Cohen, R. E. Proc. Natl. Acad. Sci. U. S. A. 2008, 105, 18200.

[55] Ellinas, K.; Tserepi, A.; Gogolides, E. Langmuir 2011, 27, 3960.

[56] Liu, M. J.; Wang, S. T.; Wei, Z. X.; Song, Y. L.; Jiang, L. Adv. Mater. 2009, 21,665 .

[57] Chen, K. L.; Zhou, S. X.; Wu, L. M. ACS Nano 2016, 10, 1386.

[58] Vogel, N.; Belisle, R. A.; Hatton, B.; Wong, T. S.; Aizenberg, J. Nat.
Commun. 2013, 4, 2167.

[59] Kang, H.; Lee, J. S.; Chang, W. S.; Kim, S. H. Adv. Mater. 2015, 27, 1282.

[60] Qiu, S.; Yin, H. C.; Zheng, J. T.; Jiang, B.; Wu, M. B.; Wu, W. T. Mater. Lett. 2014, 133, 40.

[61] Dorvee, J. R.; Derfus, A. M.; Bhatia, S. N.; Sailor, M. J. Nat. Mater 2004, 3, 896.

[62] Zhang, Y. Z.; Wang, J. X.; Huang, Y.; Song, Y. L.; Jiang, L. J. Mater. Chem. 2011, 21, 14113 .

[63] Wang, J. X.; Wang, L. B.; Song, Y. L.; Jiang, L. J. Mater. Chem. C 2013, 1,6048 .

[64] Tian, D. L.; Song, Y. L.; Jiang, L. Chem. Soc. Rev. 2013, 42, 5184

[65] Fujii, S.; Kappl, M.; Butt, H. J.; Sugimoto, T.; Nakamura, Y. Angew. Chem., Int. Ed. 2012, 51, 9809.

[66] Zhu, W.; Yang, H. W.; Lan, Y.; Yin, X. P.; Wang, S. Q.; Wang, C.; Gao, N.; Li, G. T. Adv. Mater. Interfaces 2016, 3, 1600225.

[67] Zhang, J. L.; Xue, L. J.; Han, Y. C. Langmuir 2005, 21, 5.

[68] Thompson, C. M.; Ruminski, A. M.; Sega, A. G.; Sailor, M. J.; Miskelly, G. M. Langmuir 2011, 27, 8967.

[69] Burgess, I. B.; Koay, N.; Raymond, K. P.; Kolle, M.; Lončar, M.; Aizenberg, J. ACS Nano 2012, 6, 1427.

[70] Raymond, K. P.; Burgess, I. B.; Kinney, M. H.; Lončar, M.; Aizenberg, J. Lab Chip 2012, 12, 3666.

[71] Hong, W.; Li, H. R.; Hu, X. B.; Zhao, B. Y.; Zhang, F.; Zhang, D.; $\mathrm{Xu}, \mathrm{Z}$. Chem. Commun. 2013, 49, 728.

[72] Wu, H.; Kuang, M. X.; Cui, L. Y.; Tian, D.; Wang, M. H.; Luan, G. Y.; Wang, J. X.; Jiang, L. Chem. Commun. 2016, 52, 5924.

[73] Xue, P. H.; Nan, J. J.; Wang, T. Q.; Wang, S. L.; Ye, S. S.; Zhang, J. H.; Cui, Z. C.; Yang, B. Small 2017, 13, 1601807.

[74] Wang, J. X.; Wen, Y. Q.; Feng, X. J.; Song, Y. L.; Jiang, L. Macromol. Rapid Commun. 2006, 27, 188 .

[75] Wang, J. X.; Wen, Y. Q.; Hu, J. P.; Song, Y. L.; Jiang, L. Adv. Funct. Mater. 2007, 17, 219.

[76] Wang, J. X.; Hu, J. P.; Wen, Y. Q.; Song, Y. L.; Jiang, L. Chem. Mater. 2006, $18,4984$.

[77] Ge, H. L.; Wang, G. J.; He, Y. N.; Wang, X. G.; Song, Y. L.; Jiang, L.; Zhu, D. B. ChemPhysChem 2006, 7, 575.

[78] Zhang, Y. Q.; Hao, X.; Zhou, J. M.; Zhang, Y. Z.; Wang, J. X.; Song, Y. L.; Jiang, L. Macromol. Rapid Commun. 2010, 31, 2115.

[79] Liu, J.; Jin, J.; Li, Y.; Huang, H. W.; Wang, C.; Wu, M.; Chen, L. H.; $\mathrm{Su}$, B. L. J. Mater. Chem. A 2014, 2, 5051.

[80] Xu, L.; Wang, J. X.; Song, Y. L.; Jiang, L. Chem. Mater. 2008, 20 3554.

[81] Chen, L. Y.; Lai, C. H.; Wu, P. W.; Fan, S. K. J. Electrochem. Soc 2011, 158, P93.

[82] Qin, M. M.; Li, X.; Zheng, Y. P.; Zhang, Y.; Li, C. J. Acta Chim. Sinica 2015, 73, 1161. (秦咪咪, 李昕, 郑一平, 张炎, 李从举, 化 学学报, 2015, 73, 1161)

[83] Griffete, N.; Dybkowska, M.; Glebocki, B.; Basinska, T.; Connan, C.; Maître, A.; Chehimi, M. M.; Slomkowski, S.; Mangeney, C. Langmuir 2010, 26, 11550 .

[84] Han, G. Z.; Zhu, S.; Wu, S. R.; Pang, F. F. Acta Chim. Sinica 2012, 70, 1827. (韩国志, 朱沈, 吴生蓉, 庞峰飞, 化学学报, 2012, 70 1827.)

[85] Liu, J. C.; Wan, L.; Zhang, M. B.; Jiang, K. J.; Song, K.; Wang, J. X.; Ikeda, T.; Jiang, L. Adv. Funct. Mater. 2017, 27, 1605221.

[86] Zhang, J. L.; Lu, X. Y.; Huang, W. H.; Han, Y. C. Macromol. Rapid Commun. 2005, 26, 477.

[87] Wang, T. Q.; Li, X.; Zhang, J. H.; Wang, X. Z.; Zhang, X. M. Zhang, X.; Zhu, D. F.; Hao, Y. D.; Ren, Z. Y.; Yang, B. Langmuir 2010, 26, 13715 .

[88] Du, C. G.; Cui, L. Y.; Zhang, Y. Z.; Zhao, T. Y.; Wang, J. X.; Song, Y. L.; Jiang, L. J. Nanosci. Nanotechnol. 2010, 10, 7766.

[89] Xu, L.; Li, H.; Jiang, X.; Wang, J. X.; Li, L.; Song, Y. L.; Jiang, L. Macromol. Rapid Commun. 2010, 31, 1422.

[90] Huang, Y.; Wang, J. X.; Zhou, J. M.; Xu, L.; Li, Z. R.; Zhang, Y. Z.; Wang, J. J.; Song, Y. L.; Jiang, L. Macromolecules 2011, 44, 2404.

[91] Ding, T.; Song, K.; Clays, K.; Tung, C. H. Adv. Mater. 2009, 21, 1936 .

[92] Lv, H.; Lin, Q.; Zhang, K.; Yu, K.; Yao, T. J.; Zhang, X. H.; Zhang, J. H.; Yang, B. Langmuir 2008, 24, 13736.

[93] Liu, C. H.; Ding, H. B.; Wu, Z. Q.; Gao, B. B.; Fu, F. F.; Shang, L. R.; Gu, Z. Z.; Zhao, Y. J. Adv. Funct. Mater. 2016, $26,7937$.

[94] Burgess, I. B.; Mishchenko, L.; Hatton, B. D.; Kolle, M.; Lončar, M.; Aizenberg, J. J. Am. Chem. Soc. 2011, 133, 12430.

[95] Zhong, K.; Wang, L.; Li, J. Q.; Van Cleuvenbergen, S.; Bartic, C.; 
Song, K.; Clays, K. Langmuir 2017, 33, 4840.

[96] Hong, W.; Li, H. R.; Hu, X. B.; Zhao, B. Y.; Zhang, F.; Zhang, D. Chem. Commun. 2012, 48, 4609.

[97] Sweetman, M. J.; Voelcker, N. H. RSC Adv. 2012, 2, 4620.

[98] Heo, Y.; Kang, H.; Lee, J. S.; Oh, Y. K.; Kim, S. H. Small 2016, 12, 3819.

[99] Yamaki, M.; Matsubara, K.; Nagayama, K. Langmuir 1993, 9, 3154.

[100] Denkov, N. D.; Velev, O. D.; Kralchevsky, P. A.; Ivanov, I. B.; Yoshimura, H.; Nagayama, K. Langmuir 1992, 8, 3183.

[101] Zhou, J. M.; Wang, J. X.; Huang, Y.; Liu, G. M.; Wang, L. B.; Chen, S. R.; Li, X. H.; Wang, D. J.; Song, Y. L.; Jiang, L. NPG Asia Mater. 2012, 4, e21.

[102] Kim, S. H.; Lim, J. M.; Jeong, W. C.; Choi, D. G.; Yang, S. M. Adv. Mater. 2008, 20, 3211.

[103] Kuang, M. X.; Wang, J. X.; Bao, B.; Li, F. Y.; Wang, L. B.; Jiang, L.; Song, Y. L. Adv. Optical Mater. 2014, 2, 34.

[104] Liu, M. J.; Wang, J. X.; He, M.; Wang, L. B.; Li, F. Y.; Jiang, L.; Song, Y. L. ACS Appl. Mater. Interfaces 2014, 6, 13344

[105] Bao, B.; Li, M. Z.; Li, Y.; Jiang, K. J.; Gu, Z. K.; Zhang, X. Y.; Jiang, L.; Song, Y. L. Small 2015, 11, 1649.

[106] Kawamura, A.; Kohri, M.; Yoshioka, S.; Taniguchi, T.; Kishikawa, K. Langmuir 2017, 33, 3824.

[107] Choi, S.; Jamshidi, A.; Seok, T. J.; Wu, M. C.; Zohdi, T. I.; Pisano, A. P. Langmuir 2012, 28, 3102.

[108] Ding, H. B.; Zhu, C.; Tian, L.; Liu, C. H.; Fu, G. B.; Shang, L. R.; Gu, Z. Z. ACS Appl. Mater. Interfaces 2017, 9, 11933.

[109] Qin, M.; Huang, Y.; Li, Y. N.; Su, M.; Chen, B. D.; Sun, H.; Yong, P. Y.; Ye, C. Q.; Li, F. Y.; Song, Y. L. Angew. Chem., Int. Ed. 2016, 55, 6911.

[110] Rastogi, V.; Melle, S.; Calderón, O. G.; Garcĺa, A. A.; Marquez, M.; Velev, O. D. Adv. Mater. 2008, 20, 4263.

[111] Rastogi, V.; García, A. A.; Marquez, M.; Velev, O. D. Macromol. Rapid Commun. 2010, 31, 190.

[112] Marín, Á. G.; Gelderblom, H.; Susarrey-Arce, A.; van Houselt, A.; Lefferts, L.; Gardeniers, J. G. E.; Lohse, D.; Snoeijer, J. H. Proc. Natl. Acad. Sci. U. S. A. 2012, 109, 16455.

[113] Huang, Y.; Zhou, J. M.; Su, B.; Shi, L.; Wang, J. X.; Chen, S. R.; Wang, L. B.; Zi, J.; Song, Y. L.; Jiang, L. J. Am. Chem. Soc. 2012, 134, 17053.

[114] Zhou, J. M.; Yang, J.; Gu, Z. D.; Zhang, G. F.; Wei, Y.; Yao, X.;
Song, Y. L.; Jiang, L. ACS Appl. Mater. Interfaces 2015, 7, 22644.

[115] Gu, Z. Z.; Fujishima, A.; Sato, O. Angew. Chem., Int. Ed. 2002, 41, 2067.

[116] Sun, W.; Jia, F.; Sun, Z. Q.; Zhang, J. H.; Li, Y.; Zhang, X.; Yang, B. Langmuir 2011, 27, 8018.

[117] Huang, Y.; Li, F. Y.; Qin, M.; Jiang, L.; Song, Y. L. Angew. Chem., Int. Ed. 2013, 52, 7296.

[118] Wu, L.; Dong, Z. C.; Kuang, M. X.; Li, Y. N.; Li, F. Y.; Jiang, L.; Song, Y. L. Adv. Funct. Mater. 2015, 25, 2237.

[119] Wang, Y. Z.; Wei, C.; Cong, H. L.; Yang, Q.; Wu, Y. C.; Su, B.; Zhao, Y. S.; Wang, J. X.; Jiang, L. ACS Appl. Mater. Interfaces 2016 , $8,4985$.

[120] Wang, M. H.; Meng, F. S.; Wu, H.; Wang, J. X. Crystals 2016, 6, 99.

[121] Li, B.; Jiang, B. B.; Han, W.; He, M.; Li, X.; Wang, W.; Hong, S. W.; Byun, M.; Lin, S. L.; Lin, Z. Q. Angew. Chem., Int. Ed. 2017, 56 4554.

[122] Shen, W. Z.; Li, M. Z.; Ye, C. Q.; Jiang, L.; Song, Y. L. Lab Chip 2012, 12, 3089

[123] Hou, J.; Zhang, H. C.; Yang, Q.; Li, M. Z.; Song, Y. L.; Jiang, L. Angew. Chem., Int. Ed. 2014, 53, 5791.

[124] Hou, J.; Zhang, H. C.; Yang, Q.; Li, M. Z.; Jiang, L.; Song, Y. L. Small 2015, 11, 2738.

[125] Griesebock, B.; Egen, M.; Zentel, R. Chem. Mater. 2002, 14, 4023.

[126] Zhang, J. T.; Wang, L. L.; Luo, J.; Tikhonov, A.; Kornienko, N.; Asher, S. A. J. Am. Chem. Soc. 2011, 133, 9152.

[127] Li, Y.; Qi, L. M. Acta Chim. Sinica 2015, 73, 869. (李扬, 齐利民, 化学学报, 2015, 73, 869.)

[128] Wang, W. H.; Dong, J. Y.; Ye, X. Z.; Li, Y.; Ma, Y. R.; Qi, L. M. Small 2016, 12, 1469.

[129] Li, Y.; Ye, X. Z.; Ma, Y. R.; Qi, L. M. Small 2015, 11, 1183.

[130] Cai, J. H.; Chen, S. R.; Cui, L. Y.; Chen, C. C.; Su, B.; Dong, X.; Chen, P. L.; Wang, J. X.; Wang, D. J.; Song, Y. L.; Jiang, L. $A d v$. Mater. Interfaces 2015, 2, 1400365.

[131] Cai, J. H.; Wang, T.; Wang, J. X.; Song, Y. L.; Jiang, L. J. Mater. Chem. C 2015, 3, 2445 .

[132] Wang, T.; Kuang, M.; Jin, F.; Cai, J. H.; Shi, L.; Zheng, Y. M.; Wang, J. X.; Jiang, L. Chem. Commun. 2016, 52, 3619.

[133] Wang. T.; Chen, S. R.; Jin, F.; Cai, J. H.; Cui, L. Y.; Zheng, Y. M.; Wang, J. X.; Song, Y. L.; Jiang, L. Chem. Commun. 2015, 51, 1367.

(Cheng, F.) 